ARTICLE

\title{
Differential epigenetic reprogramming in response to specific endocrine therapies promotes cholesterol biosynthesis and cellular invasion
}

\author{
Van T.M. Nguyen ${ }^{1, \star}$, Iros Barozzi ${ }^{2, \star}$, Monica Faronato ${ }^{1}, Y_{\text {Ylenia Lombardo }}{ }^{1}$, Jennifer H. Steel ${ }^{1}$, Naina Patel ${ }^{1}$,

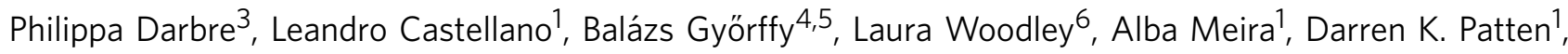 \\ Valentina Vircillo7, Manikandan Periyasamy ${ }^{1}$, Simak Ali ${ }^{1}$, Gianmaria Frige ${ }^{2}$, Saverio Minucci², \\ R. Charles Coombes ${ }^{1} \&$ Luca Magnani $^{1}$
}

Endocrine therapies target the activation of the oestrogen receptor alpha (ER $\alpha$ ) via distinct mechanisms, but it is not clear whether breast cancer cells can adapt to treatment using drug-specific mechanisms. Here we demonstrate that resistance emerges via drug-specific epigenetic reprogramming. Resistant cells display a spectrum of phenotypical changes with invasive phenotypes evolving in lines resistant to the aromatase inhibitor ( $\mathrm{Al}$ ). Orthogonal genomics analysis of reprogrammed regulatory regions identifies individual drug-induced epigenetic states involving large topologically associating domains (TADs) and the activation of super-enhancers. Al-resistant cells activate endogenous cholesterol biosynthesis (CB) through stable epigenetic activation in vitro and in vivo. Mechanistically, CB sparks the constitutive activation of oestrogen receptors alpha $(E R \alpha)$ in Al-resistant cells, partly via the biosynthesis of 27-hydroxycholesterol. By targeting CB using statins, ER $\alpha$ binding is reduced and cell invasion is prevented. Epigenomic-led stratification can predict resistance to $\mathrm{Al}$ in a subset of $E R \alpha$-positive patients.

\footnotetext{
${ }^{1}$ Department of Surgery and Cancer, Imperial College London, London W12 ONN, UK. ${ }^{2}$ IFOM-IEO Campus, European Institute of Oncology, Milan 20139 , Italy. ${ }^{3}$ School of Biological Science, University of Reading, Reading RG6 6LA, UK. ${ }^{4}$ MTA TTK Lendület Cancer Biomarker Research Group, 2nd Department of Pediatrics, Semmelweis University, Budapest H-1117, Hungary. ${ }^{5}$ MTA-SE Pediatrics and Nephrology Research Group, 2nd Department of Pediatrics,

Semmelweis University, Budapest H-1117, Hungary. ${ }^{6}$ ECMC, Charing Cross Hospital, London W120nn, UK. 7 Department of Pharmacy, Health and Nutritional Sciences, University of Calabria, Arcavacata di Rende (CS) 87036, Italy. ${ }^{*}$ These authors contributed equally to this work. Correspondence and requests for materials should be addressed to L.M. (email: I.magnani@imperial.ac.uk).
} 
O ver $70 \%$ of all breast cancers are characterized by the expression of the nuclear receptor oestrogen receptor alpha $(E R \alpha)^{1}$. Patients with $E R \alpha$-positive disease are routinely treated with adjuvant endocrine therapies (ETs) after surgery. ETs include a series of compounds designed to interfere with $\mathrm{ER} \alpha$ activation, including selective oestrogen receptor modulators (for example, tamoxifen), aromatase inhibitors (AIs, for example, Letrozole and Anastrozole) and selective oestrogen receptor downregulators (Faslodex/Fulvestrant) ${ }^{2}$. Tamoxifen competitively bind to $\mathrm{ER} \alpha$ in the place of estrogens such as estradiol (E2). AIs are designed to deplete the estrogens in circulation, while Fulvestrant irreversibly bind to ER $\alpha$ leading to $\mathrm{ER} \alpha$ degradation. Over $40 \%$ of ER $\alpha$ breast cancers eventually relapse from ETs becoming progressively refractory to further treatments ${ }^{1}$. It remains unclear whether resistance to ETs involves drug-specific mechanisms. Since ETs are characterized by different mechanisms ${ }^{2,3}$, it is conceivable that chronic exposure to therapy may impart specific selective pressure and elicit different response mechanisms to induce stable phenotypic changes.

During development and differentiation, cell identity is established by the epigenetic activation of cell-type-specific distal regulatory elements mediated by combinatorial patterns of histone modifications $s^{4,5}$. These regions are often embedded in chromatin-accessible sites and contain DNA sequence motifs for cell-type-specific transcription factors $(\mathrm{TFs})^{6}$. The epigenome retains some degree of plasticity, as cell identity can be epigenetically reprogrammed by exposure to external stimuli such as the ectopic expression of $\mathrm{TFs}^{6}$. Cancer cells can also remodel histone modifications and chromatin accessibility in response to chronic exposure to therapeutic agents ${ }^{7-9}$. For example, adaptation to oestrogen deprivation in ER $\alpha$-positive breast cancer cells invokes extensive epigenetic reprogramming especially in distal regulatory regions such as enhancers? Regulatory elements directly associated with active transcription, and more complex regulatory regions (that is, super-enhancers (SEs)) can be mapped by measuring the histone modification histone 3-lysine $27(\mathrm{H} 3 \mathrm{~K} 27 \mathrm{ac})^{10-14}$. In this study, we have investigated the nature of drug adaptation to a diverse range of ETs in isogenic ER $\alpha$-positive breast cancer cells using integrative and orthogonal epigenomic approaches.

Our data demonstrate that breast cancer cells evolve individual epigenomic and transcriptomic profiles in response to treatment. Both in vitro and in vivo, AI-resistant cells acquire the most aggressive phenotype and develop invasive characteristics in twodimensional and three-dimensional (3D). Integrative analyses of AI-resistant cells identify stable upregulation of the entire cholesterol biosynthesis (CB) pathways including genes involved in 27-hydroxyl-cholesterol (27HC) biosynthesis. 27HC stimulation is sufficient to promote oestrogen-independent $\mathrm{ER} \alpha$ binding to thousands of putative regulatory regions. In agreement with this finding, chromatin immunoprecipitation sequencing (ChIPseq) analysis of the chromatin of AI-resistant cells confirmed extensive ER $\alpha$ binding despite oestrogen-deprived conditions. Furthermore, CB blockers (statins) can reduce ER $\alpha$ binding to DNA and abrogate cell invasion. CB upregulation also occurs in vivo during breast cancer progression. Finally, we demonstrate that a CB-based signature might be used to improve the stratification of ER $\alpha$ breast cancer patients before adjuvant treatment.

\section{Results}

Adaptation to AI treatment leads to de novo invasiveness. ETs are designed to block oestrogen-driven proliferation by interfering with one specific TF (for example, ER $\alpha$ ). However, we hypothesized that the development of resistance may follow distinct routes and generate alternative phenotypes through the different molecular mechanisms specific to each agent ${ }^{2}$. To test this hypothesis, we used a series of isogenic cell lines resistant to single agents or a combination of agents (endocrine therapy (ET)resistant ETR cells, Fig. 1a) ${ }^{15}$. Our aim was to understand the connection between the acquisition of drug-resistance and breast cancer progression, particularly metastatic development. We then carried out a real-time, impedance-based assay to monitor the migratory and invasion behaviour of ETR cells. These assays demonstrated that long term estrogen deprived (LTED) cells (mimicking AI resistance ${ }^{16}$ ) specifically develop migration and invasion properties, while MCF7- and TAM/Fulvestrant-resistant cells (MCF7T and MCF7F) do not (Fig. 1b; Supplementary Fig. 1A). It is worth noting that sequential resistance did not increase these traits (LTEDT and LTEDF versus LTED). To corroborate these findings, we developed a $3 \mathrm{D}$ invasion assay (organoids assay) in which the cells were allowed to form 3D bodies and then embedded in Matrigel. In agreement with the 2D assay, AI-resistant cells (LTEDs) spread remarkably through the Matrigel environment, while the MCF7s do not (Fig. 1c; Supplementary Movie 1A-D). Finally, we developed a metastatic mouse model to validate our findings in vivo by engineering two red-fluorescent protein reporter (mCherry) lines (MCF7-FRP and LTED-RFP). After injection into the tail vein of NOD-SCID mice (Fig. 1c), only LTED cells colonize several sites including the lymph nodes and bones (Fig. 1d). Histology analyses of putative metastatic tissues from the lymph nodes and bone marrow confirms that the invading cells are of human origin and contains breast cancer protein markers ${ }^{17}$ (HNA and PBX1 (ref. 18), Fig. 1d; Supplementary Fig. 1B). Overall, these data demonstrate that AI-resistant cells acquire traits common to aggressive breast cancers. Tamoxifen- or Fulvestrant-resistant cells, although becoming resistant to the cytostatic effect of the agent, do not evolve into highly invasive cells thus supporting the notion of agent-specific reprogramming.

ETR cells follow distinct reprogramming routes. To decipher the molecular changes induced by drug-specific resistance, we developed an unbiased integrative approach combining RNA sequencing (RNA-seq) and H3K27ac ChIP-seq in ETR cells. All ETR cells express significantly lower messenger RNA (mRNA) levels for several oestrogen receptor target genes, indicating that ET treatments still exert negative pressure on $\mathrm{ER} \alpha$ signalling. As expected, MCF7F and LTEDF cells acquire an ER $\alpha$-negative status, while the rest of the panel remained $E R \alpha$ positive (Supplementary Fig. 2A). Interestingly, other chromatin components of $E R \alpha$ signalling ${ }^{19}$ (for example, ER $\alpha$ pioneer factors such as FoxA1, PBX1 and GATA3 (refs 20,21)) remain expressed at similar levels in all cell lines (Supplementary Fig. 2B,C). Cell lines with sequential resistance are characterized by a greater number of differentially regulated genes (Supplementary Fig. 3A). Correspondingly, these cells display increased numbers of potential regulatory regions as demonstrated by the significant increase in H3K27ac-positive loci (Supplementary Fig. 3B). This increase could not simply be attributed to general changes in H3K27ac distribution (Supplementary Fig. 3C).

We then carried out a gene ontology analysis on our RNA-seq data to identify potential pathways responsible for the invasive phenotype characterizing LTED cells. To increase the power of these analyses, we clustered together all the genes differentially regulated in invasive (LTED-LTEDT-LTEDF) and non-invasive (MCF7T-MCF7F) cells (Supplementary Fig. 4A). Strikingly, we found that LTED cells activated metabolic pathways and lipid metabolism (for example, super-pathway of 
a

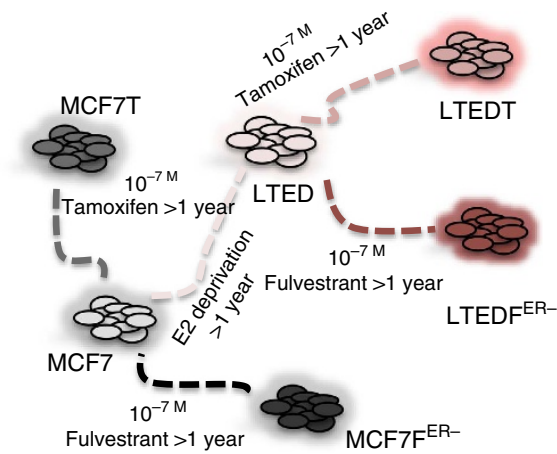

$\mathrm{ER} \alpha$ breast cancer cell adaptation protocol

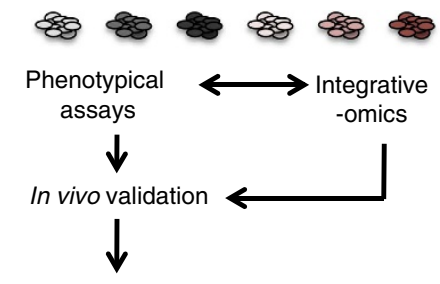

Proof of concept: biomarker/therapeutics

b
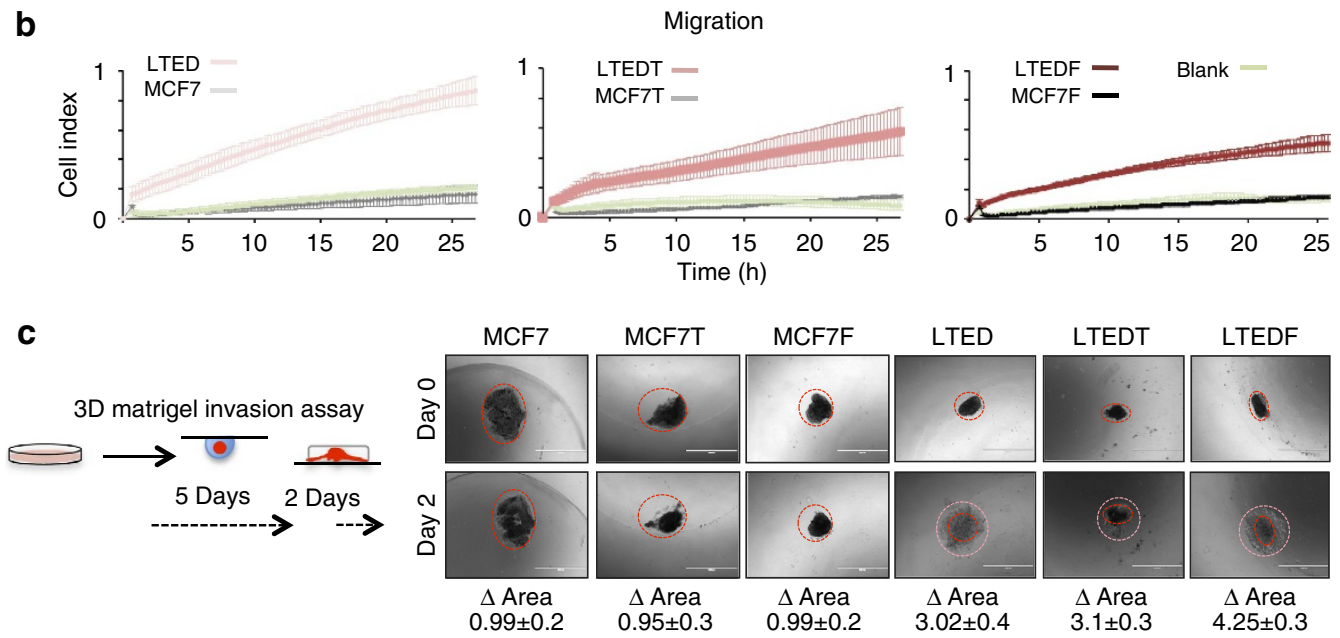

d

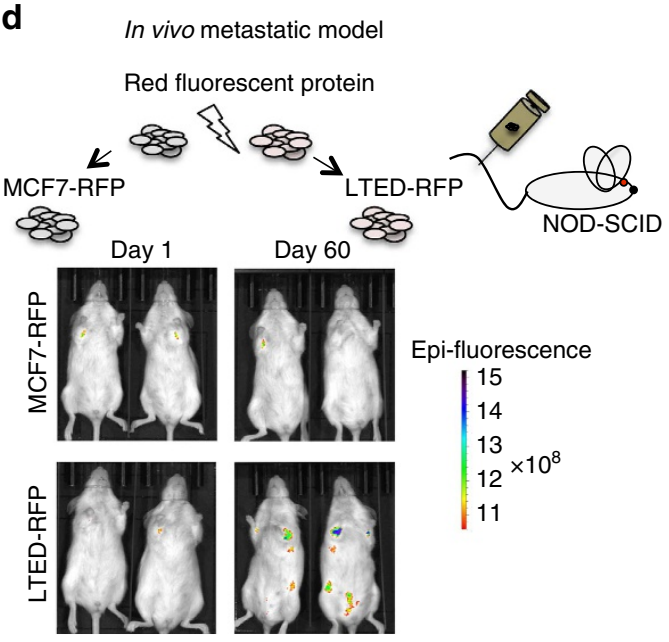

e

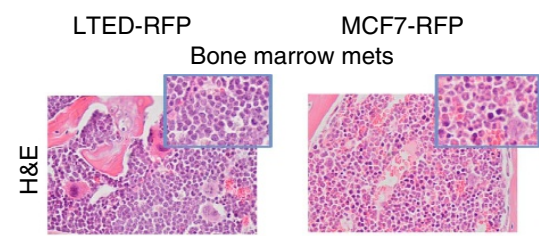

LTED-RFP
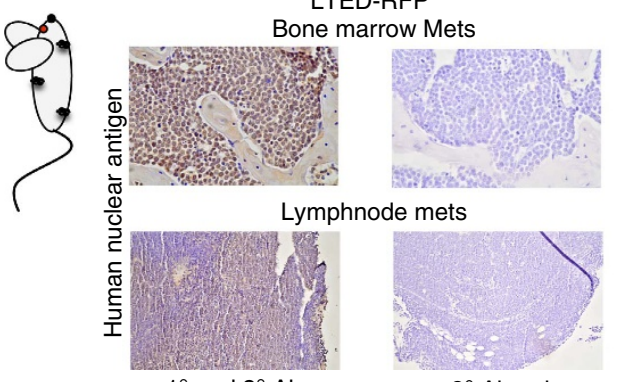

$1^{\circ}$ and $2^{\circ} \mathrm{Ab}$

$2^{\circ} \mathrm{Ab}$ only

Figure 1 | Adaptation to Al treatment is associated with cell invasion. (a) In vitro adaptation experimental protocol: ER $\alpha$-positive breast cancer cells (MCF7) were chronically exposed to endocrine therapies to generate ETR lines ${ }^{15}$. Each cell type was screened for the development of invasive potential. ETR cells were also profiled using integrative epigenomics and the results were cross-validated using clinical samples. (b) Real-time monitoring of cell migration demonstrates increased motility of LTEDs cells compared with other resistant lines. (c) 3D organoids assay was used to demonstrate invasive potential under physical constraints (Matrigel). $\Delta$ Area is calculated using ImageJ (area day 2 /area day $0, n=5$ ). The horizontal white bar indicates a distance of $1,000 \mu \mathrm{m}$. (d) LTED cells can form metastases in vivo at various sites. Cells were stably transfected with the mCherry construct, injected in the tail of the mice and followed using in vivo imaging. (e) Snapshots of histological analysis of tissues from mice injected with LTED or MCF7 cells. The first two panels represent haematoxylin and eosin (H\&E) staining on bone marrow (insets taken at a higher magnification). The bottom four panels represent immunohistochemistry on tissue from mouse bone marrow or lymph nodes showing invasion by cells immunoreactive for human nuclear antigen (left panels) or negative controls with no primary antibody (right panels).

CB, Fig. 2a; Supplementary Fig. 4B; Supplementary Data 1). More importantly, these pathways were not active in non-invasive MCF7T and MCF7F cells (grey versus red bars), suggesting differential transcriptional reprogramming (Fig. 2a). These changes are not transiently imposed by the culture condition, but rather represent stably engrained transcriptional. In point of 


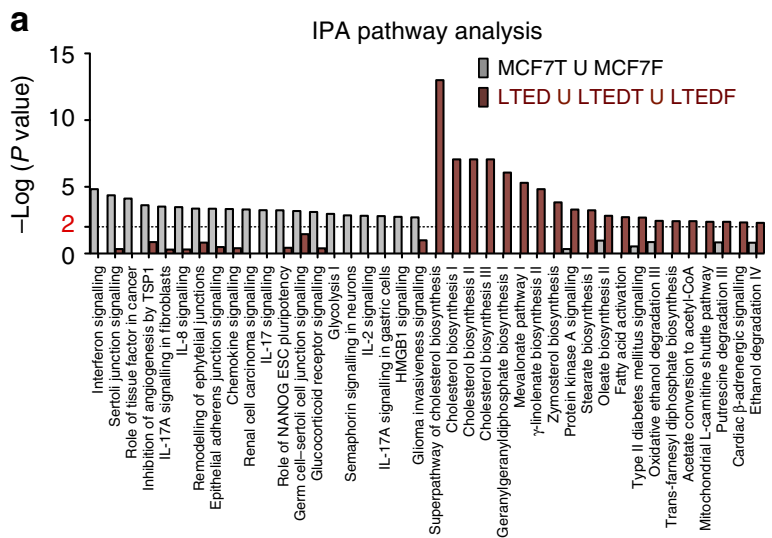

b Distal enhancer-promoter pairing strategy

C

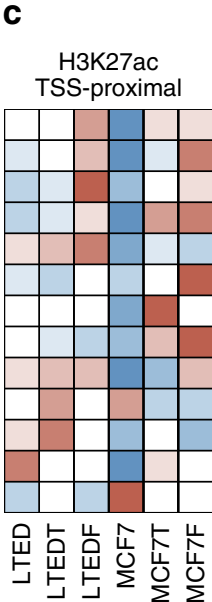

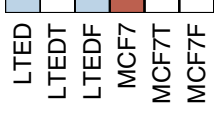

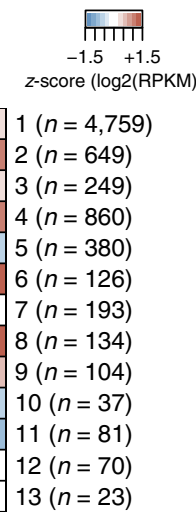

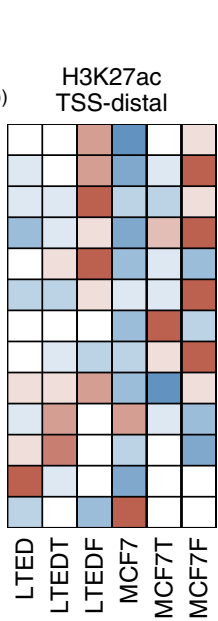

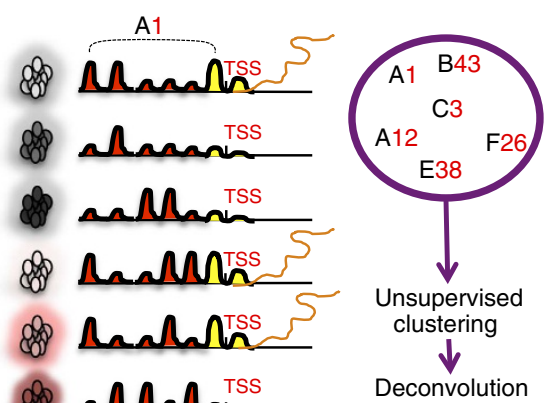

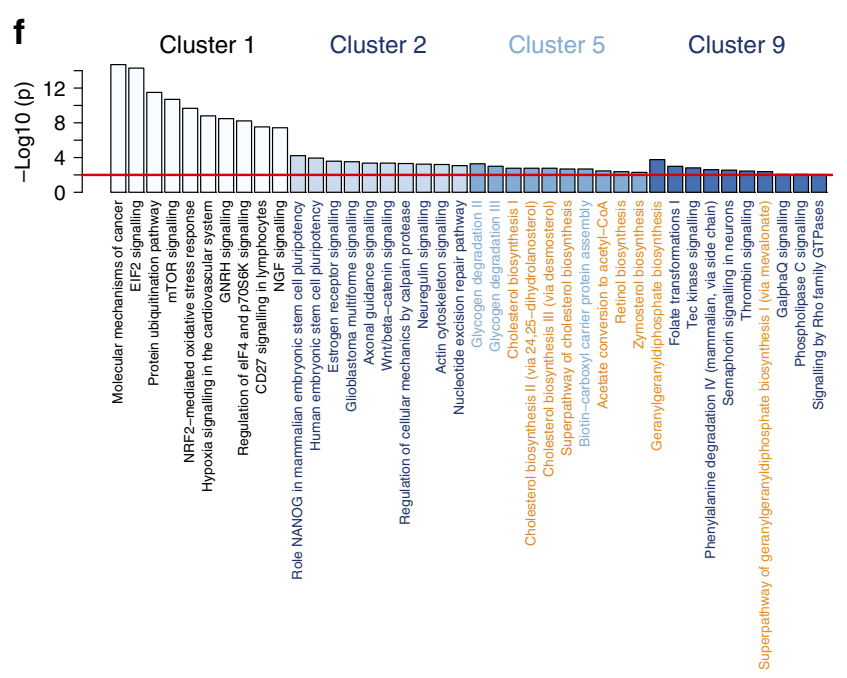

d

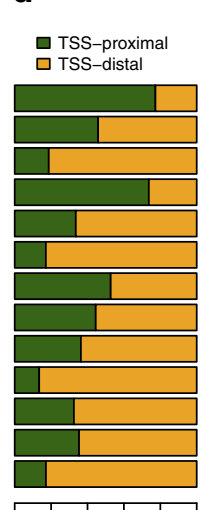

ำ $1(n=1,396)$

$2(n=767)$

$3(n=1,082)$

$4(n=307)$

$5(n=752)$

$6(n=610)$

$7(n=173)$

$8(n=167)$

$9(n=182)$

$10(n=238)$

$11(n=168)$

$12(n=128)$

$13(n=111)$

- $0000 \%$ e
e
MRNA

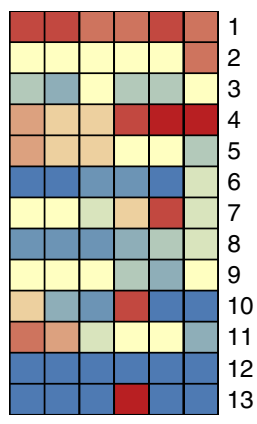

邑占㟔崩卡岕

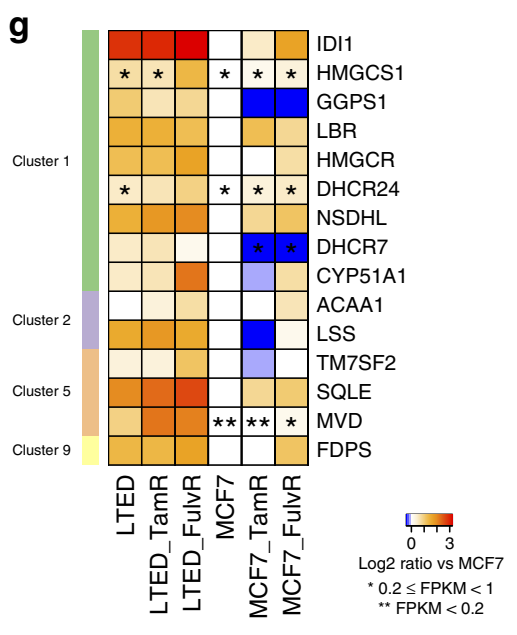

Figure 2 | Drug adaptation and epigenetic reprogramming. (a) IPA pathway analysis of differentially upregulated genes (for details of the analysis, see Supplementary Fig. 4A). The top 20 IPA pathways for invasive and non-invasive cells are plotted with their respective $P$ values. Metabolic pathways are in bold. The dotted line is set at a $\mathrm{pVal}=0.01$. (b) Diagrammatic image of the bioinformatics strategy adopted for the epigenetic analysis in the six cell lines. For more details, please see the Methods (c) Heatmaps showing the mean H3K27ac level in each cluster for each of the six cell lines. Regions were split into TSS-proximal and distal and shown as separate heatmaps. Total number of regions in brackets. (d) The proportion of TSS-proximal and distal regions in each cluster is shown. (e) Heatmap showing the median of the linear FPKM for each cluster along the cell lines. (f) IPA canonical pathways significantly enriched considering the genes putatively regulated by the TSS-proximal elements in enhancer clusters. The 10 most enriched terms were extracted and their log10 ( $P$ value) (Fisher's exact test) are shown as bar plots. The red line is set at a $\mathrm{pVal}=0.01$. ( $\mathbf{g}$ ) FPKM values of genes belonging to the 'Superpathway of Cholesterol Biosynthesis' (IPA canonical pathways) identified using H3K27ac patterns alone but also showing a significant deregulation in RNA-seq compared with MCF7. Transcripts showing low expression levels are indicated with asterisks. 
fact, we could not re-establish the original transcriptional profile (for example, MCF7) by culturing resistant cells in MCF7 culture conditions and the absence of endocrine treatment (Supplementary Fig. 4C).

We hypothesized that the reprogrammed transcriptomes evolved by invasive LTEDs cells might play a central role in driving aggressive breast cancer in vivo. To test this, we devised five individual cell signatures containing the top $10 \%$ of upregulated genes specific to each individual cell line (Supplementary Data 2) and split patients into high and low expressors (Supplementary Fig. 5A). Analysis of the METABRIC $^{22}$ data set (restricting it to the ER $\alpha$-positive patients) demonstrates that only LTED-based signatures significantly predict poor survival (Supplementary Fig. 5B,C). Collectively, these data demonstrate that AI-resistant cell lines evolve transcriptional programmes typical of patients developing more aggressive tumours.

Drug resistance invokes activation of regulatory elements. We reasoned that chronic drug exposure probably acts as a constant selective pressure within our cell line system and allow for selective activations of regulatory regions. Indeed, we demonstrated that these changes become heritable and eventually independent from media conditions. We then investigated the epigenetic circuitry that drives the cell-type-specific transcription in our ETR models. By comparing our epigenomic maps with publically available $\mathrm{H} 3 \mathrm{~K} 27 \mathrm{ac}$ profiles, we confirmed the common ancestry of ETR cells (data from ENCODE ${ }^{5}$ ), suggesting that adaptation to therapy does not entirely upset the epigenetic landscape (Supplementary Fig. 6A). Next, we devised a strategy to pinpoint the distal regulatory enhancers actively involved in regulating cell-type-specific gene expression (Fig. 2b). We first identified all the loci that showed changes in H3K27ac by comparing ETR cells with MCF7s using MACS ${ }^{23}$. We then pooled and clustered all the regions showing a significant increase or decrease in at least one cell line according to the co-variation of their patterns across the six cell lines (Fig. $2 \mathrm{~b}$ and extended Methods). Using this strategy, we extrapolate genome-wide groups of potentially interacting TSS and enhancer pairs sharing the same pattern of activity across the lines (13 different clusters). Each cluster is then de-convoluted to TSS-proximal and TSS-distal (canonical enhancers) elements (Fig. 2c,d). We then match the promoter-proximal H3K27acetylated regions to RefSeq genes and annotate the genes according to our RNA-seq analysis. These analyses demonstrate that $\mathrm{H} 3 \mathrm{~K} 27 \mathrm{ac}$ at distal enhancers is highly consistent with $\mathrm{H} 3 \mathrm{~K} 27 \mathrm{ac}$ at promoter and mRNA transcription (Fig. 2e). Of note, our analysis provides a solid alternative for studying distal regulatory regions in the absence of detailed interaction maps.

We then used Ingenuity Pathway Analysis to identify cell-typespecific pathways based on canonical enhancers (Supplementary Data 3). Cluster 1 contains mainly promoter-proximal elements (Fig. 2d) and shows an increase in H3K27ac signals in all resistant cells (Fig. 2c). In agreement, pathways significantly enriched in this cluster have been previously associated with ET resistance (mTOR, EIF2, NGF, EGF and HER2 signalling ${ }^{22,24}$ ) (Fig. 2f). Clusters 2 and 3 are directly associated to the loss of ER $\alpha$, thus demonstrating the central role of this TF in breast cancer cells. Despite over half of the regions in these groups mapping to distal enhancers, coding regions unambiguously assigned to TSSproximal elements are enriched for pluripotency genes (Fig. 2f), a feature typical of basal, stem-like ER $\alpha$-negative breast cancers ${ }^{25}$. Focusing on LTED-specific regulatory elements, cluster 5 and cluster 9 are almost exclusively enriched for genes belonging to the super-pathway of $\mathrm{CB}$ confirming our transcriptional analysis (Fig. 2f). Overall, these unbiased epigenomics analyses strongly support our initial findings, and substantiate the hypothesis that AI-resistant cells epigenetically activate distinct pathways compared with other resistant cells.

Drug-resistance-specific activation of SEs. Our integrative epigenomic analyses suggest that endogenous activation of $\mathrm{CB}$ is one of the key pathways epigenetically activated in AI-resistant cells. Epigenetic activation of the $\mathrm{CB}$ pathway appears to occur through a multi-step process. For example, many regulatory elements associated with genes belonging to $\mathrm{CB}$ are included in cluster 1 (common to all resistant cells, Supplementary Data 3), indicating that these genes are active also in Tamoxifen- or Fulvestrant-resistant cells (Fig. 2g). Nevertheless, the rate-limiting enzymes of this pathway and their associated regulatory regions (for example, HMGCR and SQLE) are ultimately activated/ upregulated only in oestrogen-deprived LTED cells (Fig. 2g). We therefore decided to investigate whether epigenomic activation of CB involves more complex epigenetic features such as SEs. Recent publications ${ }^{11,26,27}$ have identified dense clusters of distal regulatory regions that are strongly associated with transcription and particularly susceptible to perturbation, termed SEs (Fig. 3a). Adapting a previously published approach ${ }^{27}$, we identified a set of 710 SEs with different types of distribution across ETR cells (Supplementary Fig. 6B). Initial clustering analysis using SEs reveals that LTEDs cells share extensive epigenetic features. Instead, MCF7F cells maintain fewer connections with parental MCF7 further demonstrating the marked effect of ER $\alpha$ loss (Supplementary Fig. 6C). As expected, SEs activation strongly reflects cell unique traits, for example, the SEs associated with oestrogen/oestrogen receptor target gene ${ }^{17}$ EGR3 was identified only in MCF7 cells (Fig. 3a). Next, we clustered SEs to obtain 10 distinct groups using a similar approach-based on co-variance (Figs $2 \mathrm{~b}$ and $3 \mathrm{~b}$ and extended materials). IPA analysis of LTEDspecific SE clusters (clusters 3 and 5) identified once more metabolic and signalling pathways. It must be pointed out that the acetylation of SEs was strongly associated with the expression of nearby genes, in agreement with previous findings ${ }^{28}$ (Supplementary Fig. 6D; Supplementary Data 4). Finally, we identified the TF motifs buried within peak-valley-peak structures to gain further insights into the regulatory circuitry underlying these SEs. Running IPA analysis to identify pathways coordinated by TFs that could potentially bind these SE valleys, we found that LTED-specific SE clusters 3, 5 and 6 were enriched for several metabolic nuclear receptor-driven pathways (Fig. 3c, the TFs identified include FOXO1, FOXA1, FOXA2, NR5A2 for cluster 3 and RXRG, VDR, RXRB, RXRA for cluster 5). Finally, we investigated the accessible chromatin landscape to gain more detailed insights into global changes in TF occupancy using high-depth DHS-seq ${ }^{29}$. Footprinting analysis to scan for putative binding sites using DHS-seq identifies a massive increase in SREBP1 and SREBP2, the two master regulators of cholesterol homeostasis in mammals ${ }^{30}$, in LTED cells (Fig. 3d). Significantly, SREBP1/e are also the top candidates transcriptional regulators in LTED for our RNA-seq-based analysis (see Supplementary Data 1 , upstream regulators). SREBP1 activates cholesterol genes by binding their promoter after nuclear translocation in response to intracellular cues ${ }^{31,32}$. SREBP1 nuclear translocation can be inhibited using a novel series of compounds called fatostatin ${ }^{33}$. To test whether SREBP1 translocation was effectively one of the key events in CB activation, we treated MCF7 and LTED cells with fatostatin. Fatostatin treatment induced a significant reduction for several key genes in the $\mathrm{CB}$ pathway including the two rate-limiting enzymes HMGCR and SQLE specifically in LTED cells (Fig. 3e). 
a

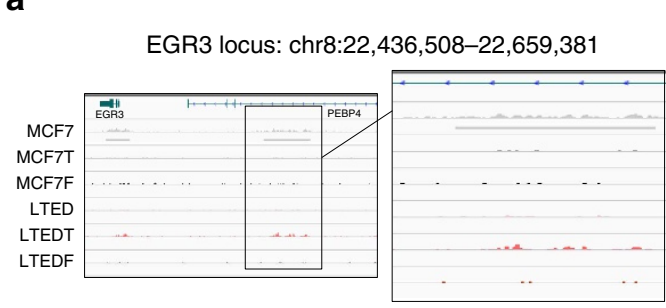

PAWR locus: chr12:79,868,240-80,072,994

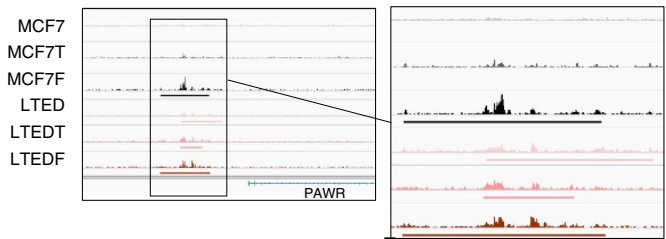

C
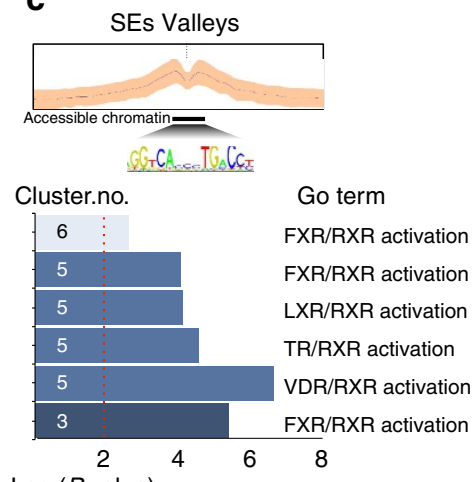

-Log ( $P$ value $)$

d b

H3K27ac

Super-enhancers

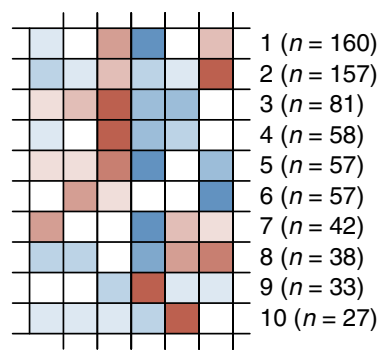

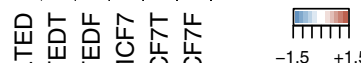

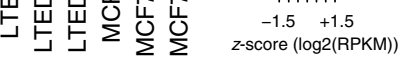

SREBPs motif in DNAsel footprints

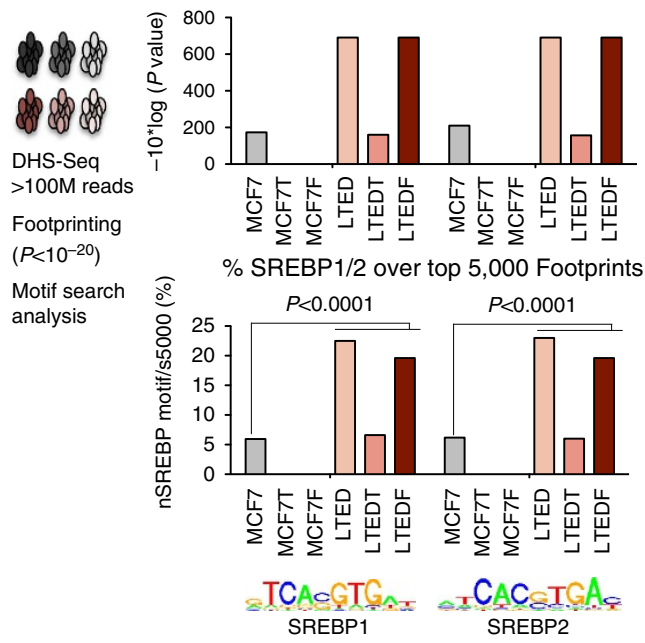

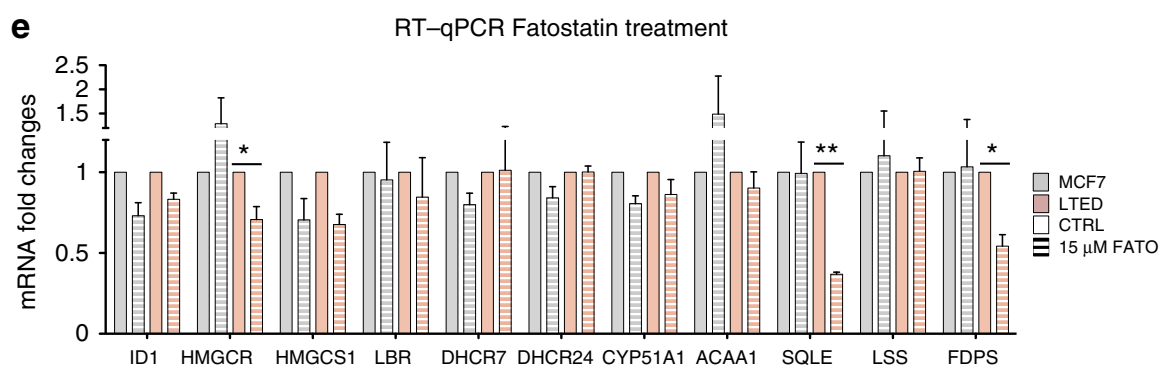

Figure 3 | Activation of super-enhancers during epigenetic reprogramming. (a) Snapshot representing SE peaks and associated raw data for two genomic locus underlying cell-type-specific phenotypes (EGR3 and PAWR). (b) Heatmaps showing the mean H3K27ac level in each cluster of SE regions for each one of the six cell lines. Total number of regions in brackets. (c) DNA motif analysis (HOMER) was carried out in the potential regulatory regions between H3K27ac-marked nucleosomes for specific clusters of SEs. Gene Ontology analysis based on potential interacting TFs is shown in the bottom panel. The vertical dashed red line indicates $P=0.01$. (d) Pipeline for the analysis of DHS-set Footprinting data sets. SREBP1 and SREBP2 footprints are uniquely enriched within the accessible chromatin landscape of LTEDs cells. The left panel indicates $P$ values from Cistrome analysis (Sitepro). The right panel displays the number of motifs within the top 5,000 footprints (expressed as a percentage). Statistical analyses were carried out using a Pearson's $\chi^{2}$-test. (e) Treatment with a SREBP1 inhibitor (Fatostatin) induces downregulation of key cholesterol biosynthesis transcripts specifically in LTED cells. Error bars represent s.e.m. calculated on three independent experiments. Asterisks indicate significant statistical differences comparing treated with mocktreated cells ( $T$-test, $P<0.05)$.

In summary, SEs and chromatin accessibility analyses further demonstrate a role for cholesterol-driven signalling centred on the potential crosstalk between nuclear receptors and cholesterolspecific TFs. These data suggest that epigenetic reprogramming might induce new transcription factor dependencies.
Epigenetic reprogramming involves larger chromatin structures. Recent evidence suggests that SEs are regulated in the context of $\mathrm{TADs}^{28}$, with relatively insulated effects within CTCF-defined regions. TADs are physically defined genomic regions that shows cohesive gene regulation during development ${ }^{33}$. Several 
studies indicate that TAD boundaries are generally resistant to differentiation ${ }^{34}$. We observed that in some instances, clusters of genes physically located in the same genomic region (for example, keratin locus, chr12q13.13) are differentially regulated as a single transcriptional unit (Supplementary Data 4). More specifically, the KRT locus (KRT7, KRT80, KRT81, KRT83 and KRT86) is upregulated in all resistant cells and its putative SE belongs to cluster 1 (Fig. 3b). This raises the possibility of coarse-grained epigenetic reprogramming occurring at the level of entire genomic compartments. To test this hypothesis, we sorted 2,283 TADs (previously identified in IMR90 cells ${ }^{33}$ ) according to the ratio of their H3K27ac levels in LTED versus MCF7 (Fig. 4a). The TAD encompassing the KRT genes (chr12:52,513,733-53,913,733) ranked in the top 5\%, showing a 1.42 -fold increase in H3K27ac in LTED cells (Fig. 4a), confirming that epigenetic reprogramming might target sets of genes simply based on their physical proximity.

We then investigated whether analogously to KRTs, CB genes are regulated in response to coarse-grain activation of their respective SE-TAD structures. Genes in the $\mathrm{CB}$ pathways were found scattered over different genomic compartments with no coherent changes in $\mathrm{H} 3 \mathrm{~K} 27 \mathrm{ac}$, suggesting a TAD-independent, punctuated regulation (Fig. 4a). Thus, while some CB genes might be passively regulated in response to widespread epigenetic changes, others may be controlled by more precise epigenetic changes, often counteracting the local TAD changes. For example, at the SQLE locus the local increase in acetylation (LTED versus MCF7) substantially overshadows the modest increase in TAD acetylation (Fig. 4b, yellow arrow). Strikingly, genes such as HMGCR and CYP51A1 gain H3K27ac signals in their putative regulatory regions despite locally losing acetylation at the TAD level (Fig. 4b, black arrows). We obtained similar results when comparing global changes in RNA transcription within the TAD to the local changes for single $\mathrm{CB}$ genes. These data strongly suggest that $\mathrm{CB}$ genes activated in non-invasive cells (Fig. $2 \mathrm{~g}$ ) might be passengers of $\mathrm{TAD}$-wide epigenetic activation, while rate-limiting $\mathrm{CB}$ genes are specifically targeted in AI-resistant cells only.

To further investigate the role of CB in AI-resistant cells, we transcriptionally profiled the entire cholesterol superpathway using reverse transcription (RT)-quantitative (q)PCR. The CB pathway is made up of 24 genes coding for enzymes necessary to build cholesterol molecules from acetyl-derived carbons (Fig. 4c; Supplementary Fig. 7A). Strikingly, 22/24 genes in the CB superpathway are upregulated in LTED cells, while 2/24 and 3/24 are upregulated in MCF7T and MCF7F cells, respectively (Fig. 4c). To rule out cell-type-specific bias in the activation of $\mathrm{CB}$, we then analysed a second ER $\alpha$-positive cell lines (in T47D breast cancer cells ${ }^{55}$ ). In agreement with MCF7 data, we observe a marked upregulation of CB transcripts in T47D-LTED cells as compared with naive T47D (21/24, Fig. 4d). On the other hand, non-tumorigenic MCF10A have much lower mRNA levels for all $\mathrm{CB}$ genes compared with non-invasive T47D or MCF7 cells (average $14 \pm 11 \%$ ).

To confirm the epigenetic nature of $\mathrm{CB}$ activation in T47D-LTED cells, we assessed the H3K27ac status of several regulatory elements comparing resistant and naive cells. In agreement with MCF7 ETR cells, T47D-LTED exhibit a strong increase in K27acetylaiton at enhancers associated with CB genes (Fig. 4e). Finally, we investigated the epigenetic activation of distal enhancers associated with $\mathrm{CB}$ directly in clinical specimens. In agreement with our in vitro data, ChIP-qPCR analysis demonstrate a significant increase in H3K27ac signal in a biopsy derived from a metastatic deposit (AI resistant) compared wih a sample derived from a primary, drug-sensitive ER $\alpha$ breast cancer patient (Fig. 4g).

Altogether, these data demonstrate that the selective pressure operated by chronic drug exposure is counteracted using very distinctive epigenetic mechanisms. More specifically, these data strongly support the centrality of $\mathrm{CB}$ epigenetic activation in AIresistant cells.

CB promotes ER $\alpha$ activation. ER $\alpha$ and other nuclear receptors can be activated by a vast array of ligands including sexual hormones (estrogens) and other cholesterol-derived compounds. Recent evidence has shown that cholesterol derivatives such as 25 and 27 hydroxycholesterol can also promote the transcription of $\mathrm{ER} \alpha$ target genes in breast cancer cells ${ }^{36,37}$. Interestingly, 27HC can also increase metastatic invasion in mice xenografted with MCF7 cells $^{38}$. We then hypothesized that one of the possible consequence of epigenetic activation of CB in AI-resistant cells might be the activation of autocrine signalling via de novo synthesis of $27 \mathrm{HC}^{39}$. To test this, we initially investigated the expression levels of CYP27A1 and CYP7B1, the two enzymes involved in the synthesis and catabolism of $27 \mathrm{HC}$ from cholesterol precursors $^{39}$. Analogously to CB genes, CYP27A1 is significantly upregulated in LTEDs compared with MCF7 and other resistant cells (Fig. 4c). Similarly, T47D-LTED exhibited an increase in CYP27A1 compared with parental T47D cells (Fig. 4d). On the other hand, we could not identify CYP7B1 transcripts in our cells (Fig. 4c,d). CYP27A1 is highly expressed in macrophages but also in ER $\alpha$-positive breast cancer cells in vivo $^{38}$. For example, we could identify strong expression of CYP27A1 in two newly established ER $\alpha$ metastatic cell line models derived from pleural effusion of AI-resistant patients (Supplementary Fig. 7B). These data suggest that LTED cells can convert the cholesterol obtained via augmented CB into $27 \mathrm{HC}$ in an autocrine pattern ${ }^{39}$.

Next, we performed ChIP-seq analysis to determine whether $27 \mathrm{HC}$ could induce $\mathrm{ER} \alpha$ chromatin binding in a way similar to other well-characterized ligands such as estradiol (E2) ${ }^{38}$ and $\mathrm{EGF}^{40}$. We performed these experiments using parental MCF7, in which endogenous $\mathrm{CB}$ is modest compared with LTED, to increase the signal to noise ratio and get robust ER $\alpha$ induction. 27HC stimulation $(1 \mu \mathrm{M})^{38}$ led to ER $\alpha$ binding to 5,174 regions. Interestingly, over $70 \%$ of $27 \mathrm{HC}$-ER $\alpha$-binding sites were occupied by E2-ER $\alpha$ or EGF-ER $\alpha$ (Fig. 5a). Shared ER $\alpha$-binding sites were enriched for H3K27Ac, H3K4me1, PBX1 and FOXA1, two TFs central to ER $\alpha$ signalling ${ }^{19}$ and were highly accessible (as shown by DHS-seq) while depleted of repressive histone marks (K9me3 and K27me3) (Fig. 5b). Shared ER $\alpha$-binding sites had over 70\% overlap with the core-ER $\alpha$-binding sites found in vivo ${ }^{41}$. We thus concluded that 27HC-bound ER $\alpha$ can be recruited to regulatory regions commonly associated with oestrogen signalling. In addition, we also identified a set of binding sites unique to 27HC activation (Fig. 5c). These sites appeared to be weaker and less enriched for K27ac but still under selective constraints (Phastcons method).

We then examined ER $\alpha$ recruitment in LTED cells cultured in the complete absence of oestrogen. We hypothesized that endogenous $\mathrm{CB}$ activity might be sufficient to promote $\mathrm{ER} \alpha$ binding to a large set of regulatory elements. Focusing on 27HC-specific ER $\alpha$-binding sites, we found that oestrogendepleted LTED cells have a higher average ER $\alpha$ binding compared with MCF7 cells grown in the same conditions (Fig. 5d). When we expanded this analysis to the entire repertoire of ER $\alpha$-binding sites (E2, EGF and 27HC in MCF7 plus LTED, total 51,974 regions), LTED cells still exhibited stronger genomewide recruitment of $\mathrm{ER} \alpha$ compared with their parental MCF7 cells grown in the absence of oestrogen (Fig. 5e), suggesting that CB activation might be sufficient to replace oestrogen in LTED cells. Blocking endogenous $\mathrm{CB}$ using Lovastatin (HMGCR inhibitor) or Terbinafine (SQLE inhibitor) induces a modest 


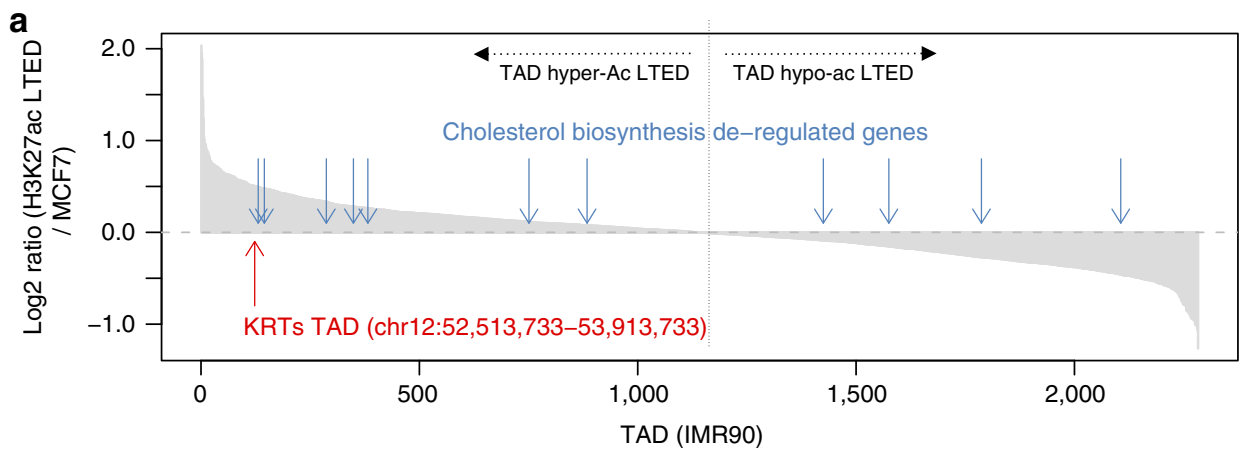

b

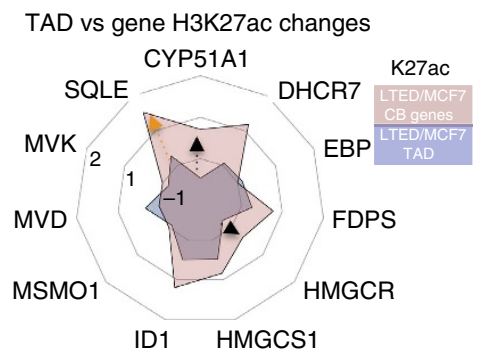

C

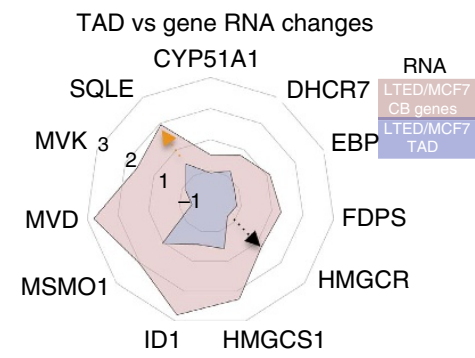

C

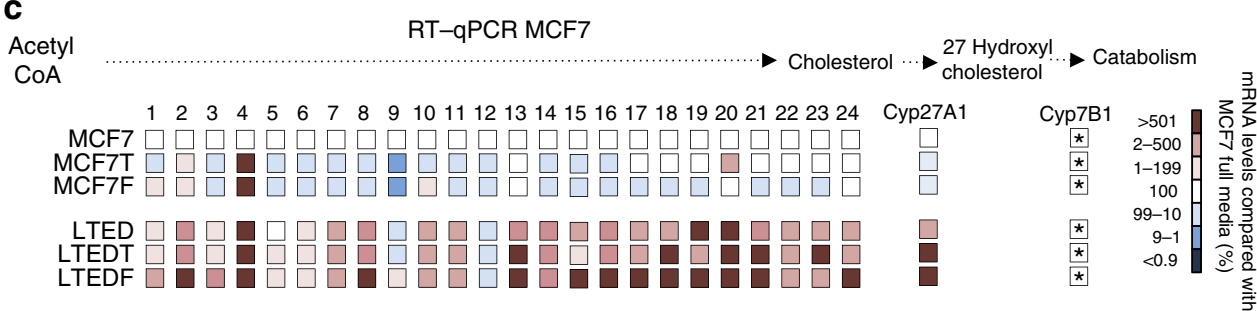

${ }^{*}$ Not expressed

d

RT-qPCR T47D

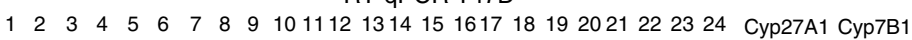
T47D-LTED
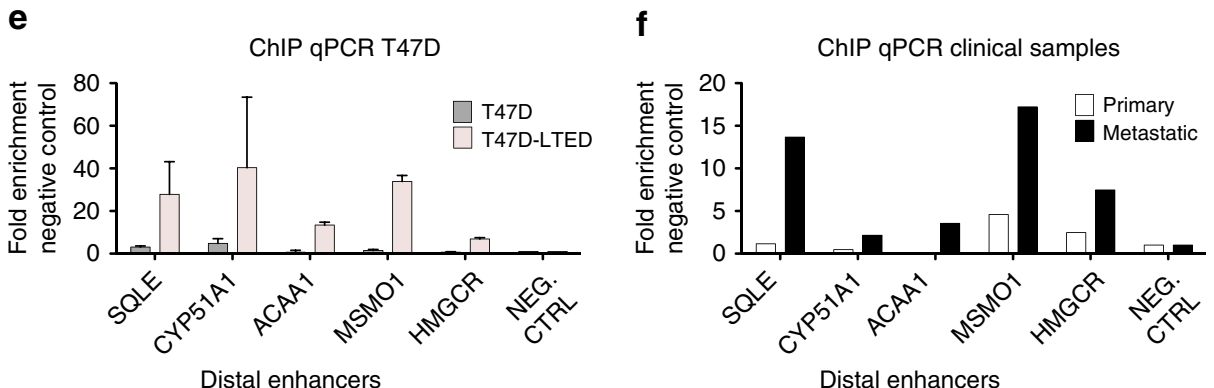

Distal enhancers

Figure 4 | Epigenetic activation of cholesterol biosynthesis. (a) In all, 2,283 topologically associating domains (TADs) (identified in IMR90 cells ${ }^{33}$ and showing acetylation in at least one out of the six cell lines) were sorted according to the ratio of their H3K27ac levels in LTED versus MCF7. The red arrow highlights the TADs encompassing the KRT genes (chr12:52,513,733 - 53,913,733) while the light-blue arrows highlights the TADs encompassing the deregulated genes in the cholesterol biosynthesis pathway. (b) Radar plots of global (TAD) versus local (CB genes) changes in H3K27ac and RNA transcription. Data are represented as ratios (Log2 RPKM LTED/RPKM MCF7). (c) RT-qPCR analysis of all the enzymes involved in cholesterol and 27HC biosynthesis (identifiers in Supplementary Fig. 8A). RNA fold differences were calculated comparing MCF7 with individual resistant cells averaging at least three independent experiments. All comparisons with different colours were statistically significant (T-test). (d) RT-qPCR analysis of all the enzymes involved in cholesterol and 27HC biosynthesis (identifiers in Supplementary Fig. 8A). RNA fold differences were calculated comparing T47D with individual resistant cells averaging at least three independent experiments. (e) ChIP-qPCR for H3K27ac demonstrates strong enrichment at distal regulatory regions controlling CB genes in Al-resistant T47D cells. Error bars represent s.e.m. calculated on three independent experiments. (f) ChIP-qPCR for H3K27ac demonstrates strong enrichment at distal regulatory regions controlling CB genes in metastatic clinical samples.

although significant reduction in $\mathrm{ER} \alpha$ recruitment at $27 \mathrm{HC}$ unique sites in LTED cells (Fig. 5f), further suggesting that CB contributes to cell-autonomous ER $\alpha$ recruitment in LTED cells.
Finally, we reasoned that epigenetic $\mathrm{CB}$ activation in AIresistant LTED cells might contribute to the invasive phenotype displayed by these cells. We therefore tested the possibility of 
a

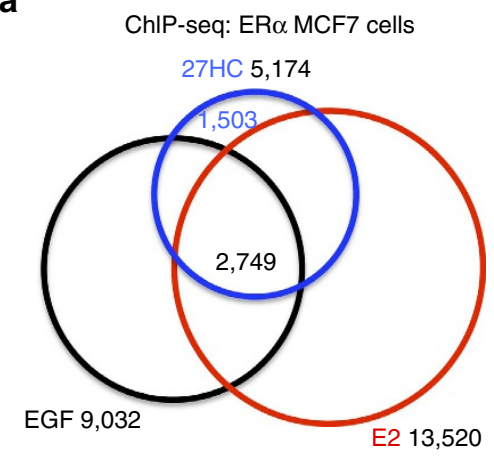

c

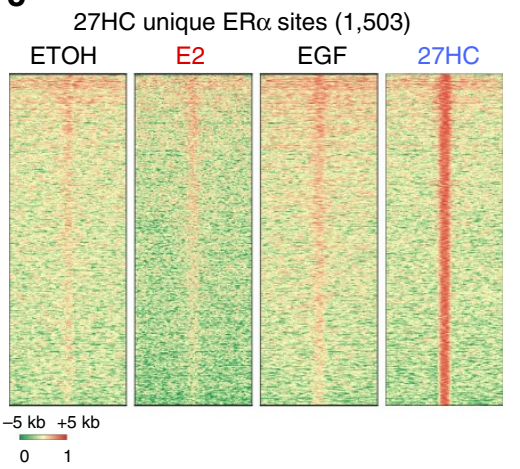

b

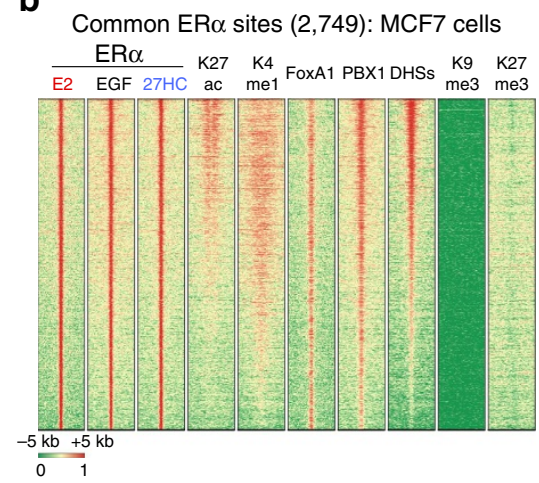

d

27HC unique $\mathrm{ER} \alpha$

e Concatenate ER $\alpha$ - ERa ETOH LTED

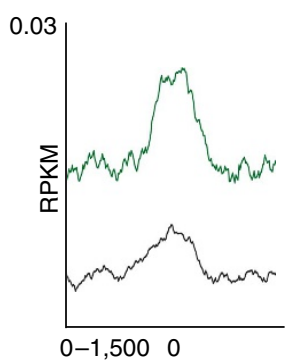
- ERa ETOH MCF7

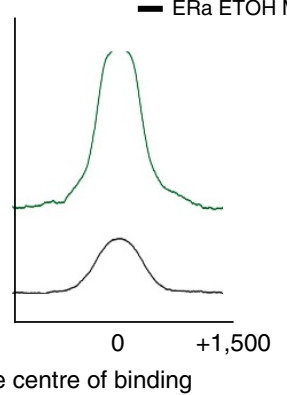

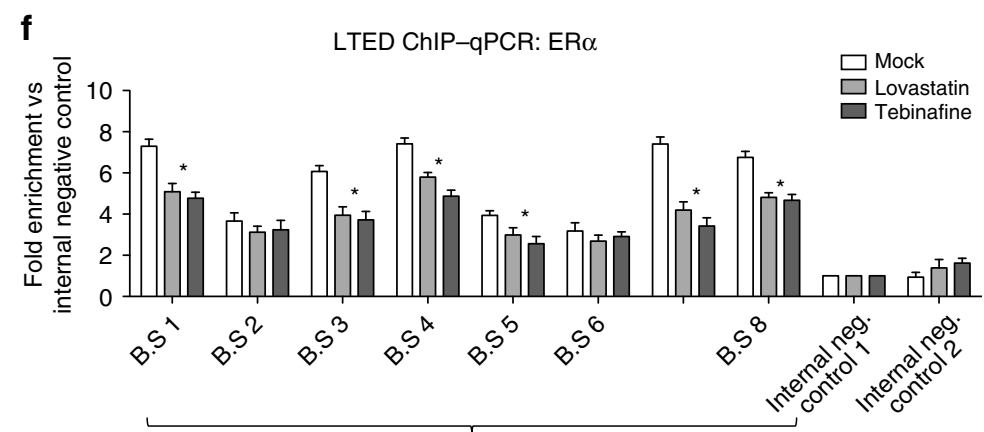

27HC-specific ER $\alpha$-binding sites

Figure 5 | 27-Hydroxycholesterol promotes ER $\alpha$ binding. (a) Venn diagrams of ligand-specific ER $\alpha$ cistromes. (b) Heatmaps showing the epigenetic features of ER $\alpha$-binding sites common to all stimuli. (c) Heatmaps showing ER $\alpha$ binding at 27HC-specific loci. (d) Comparison between ER $\alpha$ ChIP-seq signals from LTED (green) and MCF7 (black) grown in the absence of estrogens at 27HC-specific sites. (e) Same analysis as in $\mathbf{d}$ focusing on all ER $\alpha$-binding sites (right panel). (e) ChIP-qPCR analysis shows reduced ER $\alpha$ binding at 27HC-specific ER $\alpha$-binding sites upon treatment with CB pathway inhibitors ( $48 \mathrm{~h}$ treatment). Error bars represent s.e.m. calculated on three independent experiments. Asterisks indicate significant differences compared with mock-treated cells (analysis of variance, with Dunnet's $t$-test $P<0.05$ ).

blocking cellular invasion by targeting CB. As expected, LTED cells tended to be more sensitive to inhibition of $\mathrm{CB}$ as demonstrated by their respective half-maximal inhibitory concentration ( $\mathrm{IC}_{50}$ ) (MCF7: 89 and $90 \mu \mathrm{M}$, LTED: 22 and $42 \mu \mathrm{M}$, Lovastatin and Terbinafine, respectively). More importantly, inhibition of $\mathrm{CB}$ or short interfering RNA-mediated depletion of SQLE was sufficient to block the invasion of AI-resistant LTED cells in 3D Matrigel assay (Fig. 6a; Supplementary Movie 5A-D). Conversely, treatment with mevalonate (the byproduct of HMGCR) significantly increased the invasive potential of noninvasive MCF7 cells (Fig. 6b). SQLE depletion also resulted in the significant repression of several genes involved in cellular invasion (Fig. 6c). Altogether, these data strongly suggest that epigenetically activated $\mathrm{CB}$ contributes to the invasive phenotype exhibited by AI-resistant LTED cells.
CB serves as a biomarker in AI-treated breast cancer patients. Our integrative analysis of breast cancer cell lines resistant to individual ETs identified epigenetic activation of $\mathrm{CB}$ as a potentially important resistance mechanism specific to AI-treated patients. We therefore investigated whether our findings had translational potential and could be used to stratify patients before endocrine treatment. We initially developed a gene signature (Supplementary Data 1) to retrospectively stratify TCGA ER $\alpha$-positive breast cancer patients that expressed high or low levels of CB gene mRNA levels. We used TCGA data since it was possible to retrieve treatment information from these patients (AI $n=144$, Tamoxifen $n=127$ ). ER $\alpha$-positive patients with high expression of our CB-based signature at diagnosis consistently display shorter recurrence- and metastatic-free survival and are characterized by poor survival (Supplementary Fig. 8A,B). The 
a
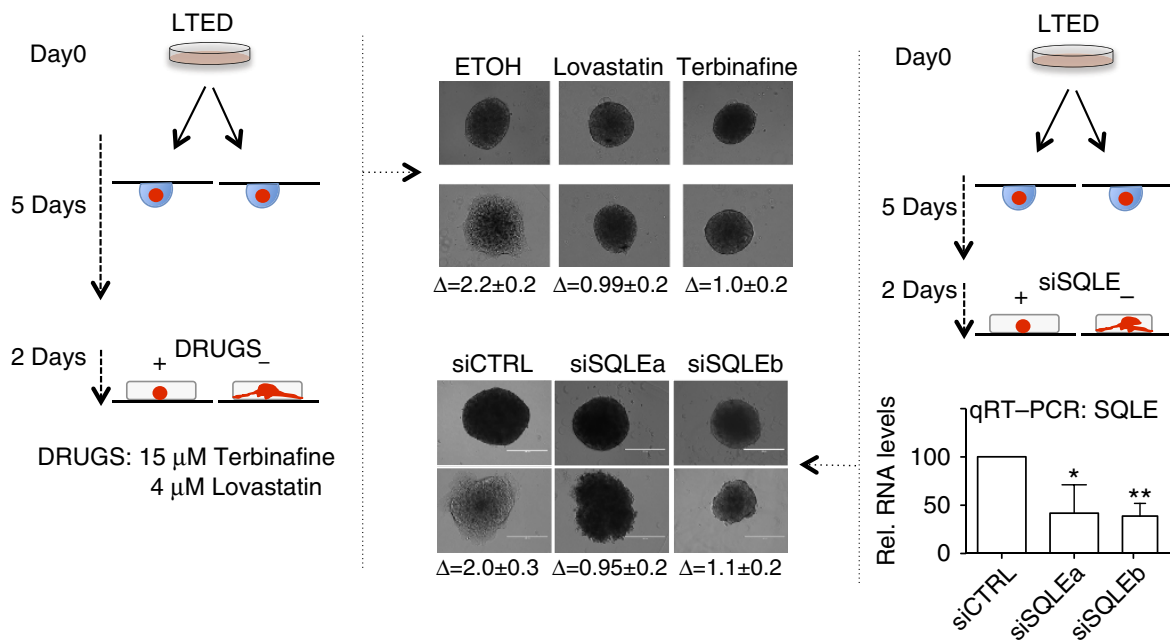

b

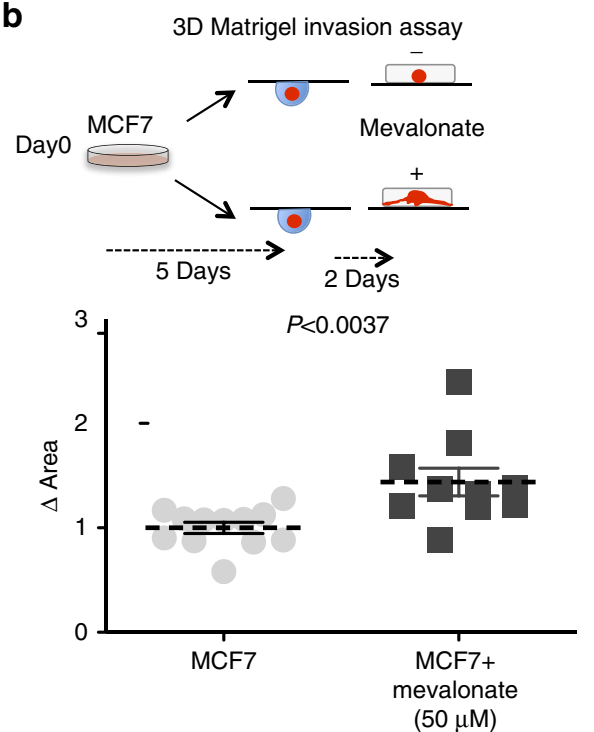

C
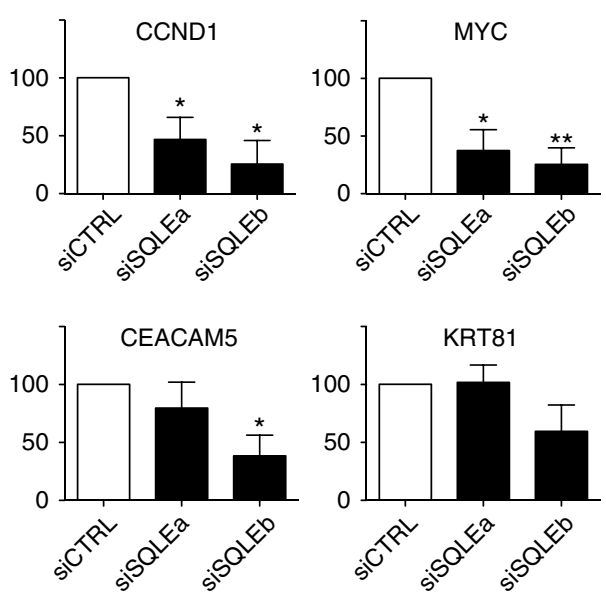

Figure 6 | Blocking cholesterol biosynthesis reduces cellular invasion. (a) Blocking cholesterol biosynthesis via treatment with CB inhibitors (left panel) or direct depletion of SQLE (right panel) is sufficient to arrest LTED invasion in Matrigel organoids assays. The horizontal white bar indicate a distance of $400 \mu \mathrm{m}$. $\triangle$ Area is calculated using Image (area day 2 /area day $0, n=5$ ). SQLE RNA fold changes were determined comparing two independent short interfering RNA ( $\operatorname{siRNA})(\mathbf{a}, \mathbf{b})$ versus a non-targeting control. Bars represent the mean with the s.d. of three independent experiments. Asterisks indicate significant difference (analysis of variance (ANOVA), $P<0.05$ ). (b) Treatment with Mevalonate increases the invasive potential of MCF7 cells in 3D organoid assays. $\Delta$ Area is calculated using ImageJ (area day 2/area day 0). $P$ value was calculated using a Student's $T$-test. (c) qRT-PCR analysis of genes involved in invasion/migration in LTED cells upon SQLE depletion. KRT81 is shown as a control. RNA fold changes were determined comparing two independent siRNAs versus a nontargeting control. Error bars represent s.e.m. calculated on three independent experiments. Asterisks indicate significant differences (ANOVA, $P<0.05$ ).

same signature cannot be used to identify $\mathrm{ER} \alpha$-negative patients, suggesting a significant degree of specificity (Supplementary Fig. $8 \mathrm{C}$ ). There are no significant changes in performance when correcting for luminal A or luminal B (Supplementary Fig. 9). CB can be directly upregulated in response to p53 mutations ${ }^{42}$; however, MCF7 cells are p53 $3^{\text {wt }}$ and LTED do not acquire p53 mutations. On the other hand, T47-LTED incur in significant CB activation despite $\mathrm{T} 47 \mathrm{D}$ being already $\mathrm{p} 53^{\mathrm{mut}}$, suggesting a degree of independence between the genetic status of p53 and the activation of CB. Finally, we still observed a strong prognostic significance within the ER $\alpha$-p53-mutated subpopulation, suggesting the existence of additional mechanisms (Supplementary Fig. 9).

Most importantly, our CB signature was particularly efficient in assessing overall survival in AI-treated patients but not in
Tamoxifen-treated patients (Fig. 7a) again suggesting that endocrine resistance is driven by distinct mechanisms. Examining the expression of genes involved in $\mathrm{CB}$ in an independent cohort (FEMARA trial $^{43}$ ), we find that MSMO1, MVD and SQLE mRNA levels are statistically higher in patients that did not respond to neo-adjuvant (pre-surgery) AI therapy (Fig. 7b). Overall, these data suggest that patients with high CB are less likely to benefit from AI treatment and are in substantial agreement with our in vitro results.

SQLE is the second rate-limiting enzyme in the CB pathway and one of the genes epigenetically activated specifically in LTED cells (Figs $2 \mathrm{~g}$ and $4 \mathrm{~b}$ ). As expected, SQLE-encoded protein is strongly upregulated in all LTED models (Fig. 7c). We therefore quantified mRNA levels for HMGCR and SQLE in an $\mathrm{ER} \alpha$ breast cancer patient treated with AI for whom we had 
a

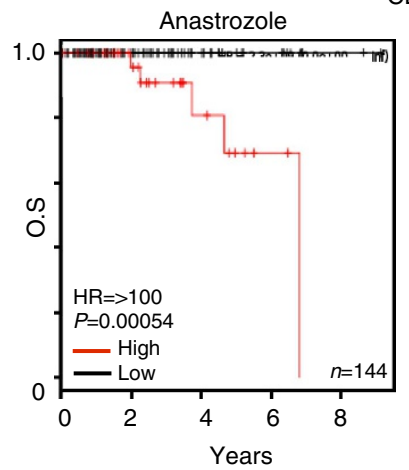

b

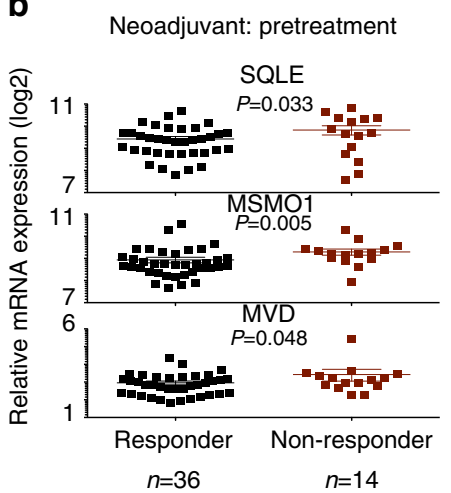

CB signature TCGA

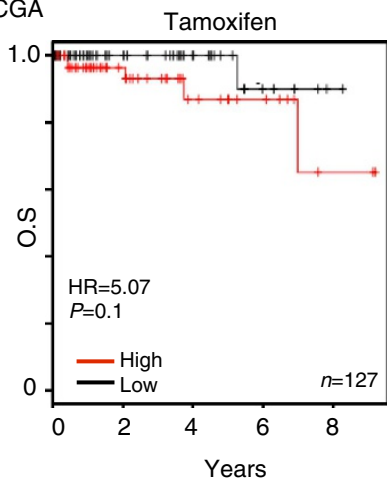

\section{C}
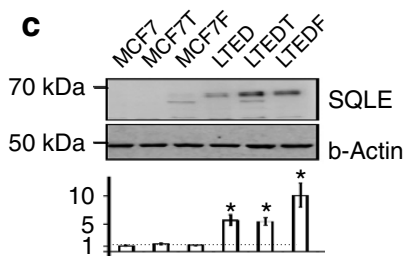

d

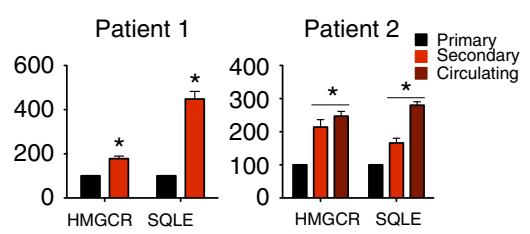

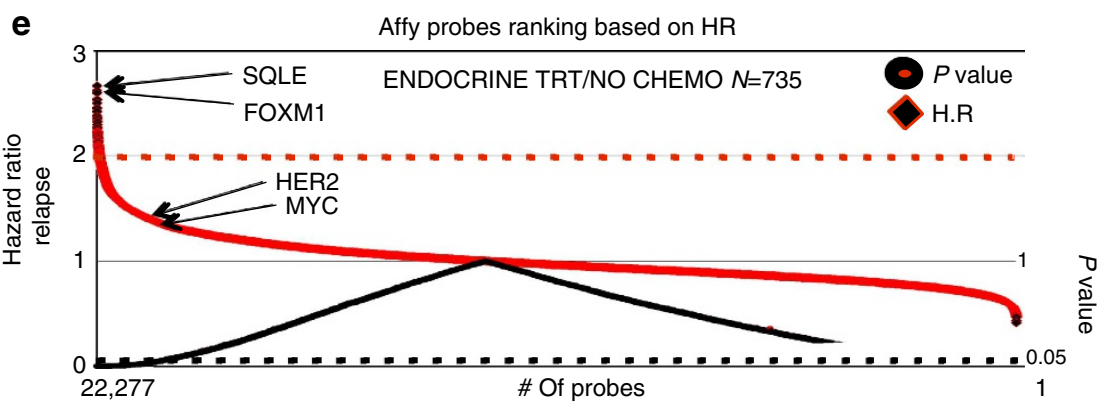

Figure 7 | CB-guided stratification of Al-resistant patients. (a) Kaplan-Meier survival plots using averaged expression of differentially regulated enzymes (RNA-seq) involved in cholesterol biosynthesis in specific subgroups of ET-treated patients. (b) Pretreatment mRNA levels (FEMARA Trial, see text) of key enzymes can help identify Al-resistant patients. P values were calculated using a Mann-Whitney U-test. (c) Western blot analysis of SQLE and protein quantification. Error bars represent s.e.m. calculated on four independent experiments. Asterisks indicate significant difference comparing each cell type with MCF7 cells (analysis of variance, $P<0.05$ ). (d) HMGCR and SQLE mRNA levels were quantified in longitudinal samples from breast cancer patients. mRNA was extracted from FFPE slides marked by a registered pathologist. Error bars represent s.e.m. calculated on three independent experiments. Asterisks indicate significant differences compared with primary samples ( $T$-test, $P<0.05$ ). (e) Manhattan plot for the prognostic potential of all human genes contained in the affy-chip U133. Both hazard ratio and $P$ values are represented. Red and black dotted lines represent hazard ratio $=1$ and $P<0.05$, respectively.

longitudinal data (RNA obtained at diagnosis, first relapse and in circulating metastatic cells from pleural effusion). In agreement with our previous finding, we found that key $\mathrm{CB}$ genes are progressively upregulated during cancer progression (Fig. 7d). Overall, SQLE emerged from these analyses as a novel candidate biomarker to identify AI-resistant breast cancer patients. This was further confirmed by our unbiased analysis of the entire human transcriptome. When we sorted all 22,277 probes present in the U133A gene chip according to their potential to predict relapse in a panel of $724 \mathrm{ER} \alpha$-positive breast cancer patients treated with ET, SQLE (probe set 209218_at) ranked 1st (hazard ratio $=2.67$ ) (Fig. 7e; Supplementary Data 5). Finally, we speculated that genetic-epigenetic interactions might facilitate SQLE expression in ER $\alpha$-positive breast cancer. Remarkably, SQLE is amplified in almost $10 \%$ of $\mathrm{ER} \alpha$-positive patients and overexpressed in an additional $13 \%{ }^{44}$ (Supplementary Fig. 10A). SQLE amplification directly correlates with increased mRNA expression in primary tumours (Supplementary Fig. 10B). SQLE emerges also as one of the top-ranking overexpressed genes in 10 breast cancerindependent data sets (invasive ductal carcinoma (IDC) versus normal breast, Supplementary Data 6). More importantly, SQLE amplification or mRNA overexpression is sufficient to stratify outcome in ER $\alpha$-positive breast cancer patients from the TCGA cohort (Supplementary Fig. 10C). Altogether, our data strongly support the prediction generated using drug-specific resistant cells and confirm a functional role for epigenetic activation of $\mathrm{CB}$ as a mechanism of resistance to $\mathrm{AI}$ therapy in vivo. 


\section{Discussion}

In addition to clonal selection ${ }^{41}$, adaptation to drug treatment may involve heritable transcriptional changes that allows cells to survive in the presence of externally imposed selective pressure ${ }^{7,8}$. Using ER $\alpha$-dependent breast cancer cells as a working model, we demonstrate that adaptation to therapeutic agents sharing the same target follow distinct routes depending on the specific mechanism of action. More specifically, the appearance of oestrogen-independent growth corresponds to the epigenetic activation of $\mathrm{CB}$. $\mathrm{CB}$ functionally contributes to the invasive phenotype AI-resistant cells and $\mathrm{CB}$ activation can be used to predict resistance to AI before treatment. Drug-resistance, however, does not invariably coincide with the development of more aggressive phenotypes, suggesting that in some instances the transition to metastatic progression requires additional reprogramming. Using recently developed paradigms, our data demonstrate that individual drugs impose very specific epigenetic changes that target both local regulatory elements such as individual enhancers and large topological domains and SEs ${ }^{11}$. This is surprising considering that endocrine treatments impinge on the same TF (for example, ER $\alpha$ ). It will be important to evaluate whether a similar process occurs also in other malignancies.

TF motif analyses of epigenetically reprogrammed loci show that deregulation of the core network of TFs, which lies at the basis of cell-type specification (for example, FoxA1) ${ }^{45}$ might lead to aberrant rearrangements of entire chromosomal territories. These rearrangements may include surrounding genes that are central to functional reprogramming, drug resistance and invasion. At the same time, specific selective pressures might trigger epigenetic activation of tailored pathways (for example, $\mathrm{CB})$, irrespective of the broad chromatin context in which single genes are nested.

Our data support a model in which chronic exposure to AI constitutively activates $\mathrm{CB}$. Intra-tumour activation of $\mathrm{CB}$ may then lead to the local accumulation of metabolic ligands for other nuclear receptors. Promisingly, we find that AI-resistant SEs are enriched with DNA motifs of metabolic nuclear receptors including FXR and LXR. It is well established that 27HC is also an LXR agonist ${ }^{38}$, therefore we cannot exclude that LXR might also contribute to AI resistance in combination with endogenous $\mathrm{ER} \alpha$ activation $^{46}$. In addition, endogenously produced ligands, including $27 \mathrm{HC}$ might act on additional nuclear receptors and allow the activation of an alternative cohort of regulatory elements (Supplementary Fig. 11). Of note, 40/48 nuclear receptors (NRs) have increased expression in LTED cells (FXR $>300$ fold, LXR $>1.8$ fold) and 9 of these are markedly increased in ER $\alpha$-negative LTEDF (PPAR $\gamma>500$-fold) strongly suggesting that other nuclear receptors might contribute to the invasive phenotype observed in LTEDF cells. In the same way, autocrine metabolic signalling might also contribute to $\mathrm{ER} \alpha$ binding found in metastatic breast cancer in vivo ${ }^{41}$.

Epigenetic activation of the $\mathrm{CB}$ pathway might also be supported by specific genetic aberrations. For example, SQLE amplification occurs in a significant proportion of cancers ${ }^{47}$, but the functional consequences of SQLE amplification have not previously been appreciated. SQLE locus is just $2 \mathrm{Mb}$ away from the oncogene $M y c$ in a hotspot for genomic rearrangements ${ }^{44}$. However, we could not find any clear correlation between SQLE and MYC expression, suggesting that the poor outcome typical of breast cancer patients with high SQLE does not simply reflect MYC activation. Notably, our model could partially explain recently published epidemiological observations. While the protective role of anti-cholesterol statin towards breast cancer is still debated, a nation-wide study found that statin users with $\mathrm{ER} \alpha$-positive breast cancer are less likely to develop relapses ${ }^{48}$. In agreement with our findings, no effect on relapse rates was noted in patients with $\mathrm{ER} \alpha$ negative ${ }^{48}$

Recent clinical trials recommend a switch from Tamoxifen to AI with ovarian suppression in pre-menopausal women ${ }^{49}$. Our data suggest that Tamoxifen and AI exposure do not have an equivalent effect on breast cancer cells; thus ER $\alpha$ patients should be assigned to a specific endocrine treatment depending on the molecular profile of their tumour. Moreover, our data offer novel molecular insights and warrants for additional clinical trials combining statins and $\mathrm{AI}$ in patients with metastatic disease ${ }^{47}$. Pharmacodynamics studies show no influence of statins on E2 levels or Anastrozole metabolism ${ }^{50}$. Interestingly, statins were shown to interfere with ovarian cancer and multiple myeloma growth in pre-clinical models ${ }^{51,52}$. Our work therefore offers a new paradigm in the context of how epigenomes evolve in response to interrelated exogenous pressures while paving the way for future studies designed to help us understand the specific effects of therapeutic intervention during metastatic progression.

\section{Methods}

Cell lines and clinical samples. Cell lines were cultured as follows MCF7 and T47D: DMEM cell growth medium (DMEM with $10 \%$ of fetal bovine serum (FBS) and $100 \mathrm{U}$ penicillin $/ 0.1 \mathrm{mg} \mathrm{ml}^{-1}$ streptomycin plus $10^{-8} 17$ - $\beta$-estradiol (SIGMA E8875). LTED cells were passaged in stripped DMEM cell growth medium (Phenol-free DMEM with 10\% of charcoal-stripped FBS (CDT) and $100 \mathrm{U}$ penicillin/0.1 $\mathrm{mg} \mathrm{ml}^{-1}$ streptomycin MCF7T and LTEDT were cultured as MCF7 and LTED with the addition of $10^{-7}$ 4-hydroxytamoxifen (SIGMA H7904). MCF7F and LTEDF were cultured as MCF7 with the addition of $10^{-7}$ Fulvestrant (SIGMA $\mathrm{I}_{4409)^{15}}$. Tissues (primary, secondary and pleural effusions) were obtained from the Imperial Tissue Bank. The Tissue Management Committee reviewed and approved all documents. Imperial Tissue bank released stores the samples under an HTA Licence (12275). The appropriate regulatory committees approved all the relevant documents. Each patient signed an informed consent to donate tissue to Imperial Tissue Bank. All patients' notes are kept within locked filing cabinets within locked offices in research. This is to maintain confidentiality. Tissues used for qRT-PCR were manually enriched for tumour cells by the resident pathologist. Pleural effusions were cultured in DMEM, 10\% FBS and phenol for a brief time before RNA extraction. Cells were tested, genotyped and characterized as ER $\alpha$ positive (LGC standards). Circulating tumour cells were characterized as mammary gland based on short tandem repeat similarity with HTB-22 (LGC standards).

Invasion assays. Boyden chamber and 3D Matrigel invasion assays were performed as previously described ${ }^{53}$. Briefly, cells $(5 \times 104$ in $200 \mu$ of $\alpha$-MEM) were plated in the Matrigel-coated upper chambers of the 24-well Transwell invasion assay plate (Corning). Each condition was represented in triplicate. Plates were incubated at $37^{\circ} \mathrm{C}$ for $24 \mathrm{~h}$ for HCC1806 cells. Cells in the lower chamber (including those attached to the under surface of the membrane) were trypsinized and counted using a Casy 1 counter (Sharfe System). Mean and s.e.m. of independent experiments were calculated. Statistical analysis was performed using a two-tailed Student's $t$-test to determine the statistical significance of the differences observed. A $P$ value below 0.05 was considered significant.

Fatostatin and mevalonate treatments. Cell lines were cultured in the appropriate conditions until $60-70 \%$ confluence and then treated with $15 \mu \mathrm{M}$ of Fatostatin (SIGMA F8932) for $6 \mathrm{~h}$ before RNA extraction and qRT-PCR analysis. For mevalonate treatment, organoids were allowed to form and embedded in Matrigel (see Hanging-drop assay) then treated with $50 \mu \mathrm{M}$ mevalonate ${ }^{54}$ (SIGMA 90469).

Mouse models. MCF7-mCherry-Puro and LTED-mCherry-Puro cells $\left(1 \times 10^{6}\right)$ were injected into the tail vein of NOD-SCID mice ( $n=3$ per group). Images were taken of the mice weekly from dorsal and ventral views for 8 weeks to monitor the development of metastases. The mice were assessed weekly using whole-body imaging to quantify the relative amounts of tumour burden. Imaging was performed with a highly sensitive, cooled charge-coupled device camera mounted in a light-tight specimen box (IVIS; Xenogen). The imaging and quantification of signals were controlled by acquisition and analysis software. At termination, the lymph nodes, lungs, kidney, bones and livers were harvested by dissection. The lymph nodes, lungs, kidneys, liver and bones were fixed for $24 \mathrm{~h}$ in neutral buffered formalin, and then rinsed in $70 \%$ ethanol. Following fixation, the bones were decalcified in $10 \%$ EDTA in $0.1 \mathrm{M}$ Tris- $\mathrm{HCl} \mathrm{pH} 7.4$ for 1 week, re-fixed in formalin and then rinsed in $70 \%$ ethanol. All tissues were dehydrated using ethanol, cleared with Histoclear and embedded in paraffin wax. Paraffin sections $(5 \mu \mathrm{m})$ were 
stained with haematoxylin and eosin to reveal their tissue morphology and the presence of infiltrating cells. The animal trials were carried out under London Home Office license authority and London Home Office Ethics Committee guidelines.

Immunohistochemistry and western blot. Paraffin sections were dewaxed and antigen retrieval was carried out with citrate buffer at $\mathrm{pH} 6.0$ or in $10 \mathrm{mM}$ Tris-HCl, $\mathrm{pH}$ 9.0. Tissue sections were pretreated using $0.3 \% \mathrm{H}_{2} \mathrm{O}_{2}$ in PBS, rinsed in PBS and then incubated with $20 \mu \mathrm{l} \mathrm{ml}^{-1}$ normal goat serum. Primary antibodies were diluted in PBS and incubated overnight at $4{ }^{\circ} \mathrm{C}$, along with negative control sections where the primary antibody was omitted. All the bound antibodies were detected using biotinylated anti-mouse or anti-rabbit secondary antibodies, detected using the Vectastain Elite peroxidase $\mathrm{ABC}$ kit and the ImmPACT DAB kit (Vector Laboratories). Stained sections were counterstained with haematoxylin. Antibody dilutions: PBX1 1:50 (Abnova H00005087-M01), Human nuclear antigen 1:25 (Abcam ab191181), Pan-cytokeratin 1:100 (SIGMA C5992). For western blot, SQLE antibody (SIGMA HPA018038) was used at 1:250 and ER $\alpha$ (Santa Cruz sc-543) was used at 1:1,000.

Hanging-drop assay. Briefly, cells were trypsinized and counted. In all, 250,000 cells were resuspended in $1 \mathrm{ml}$ of phenol-red or phenol-red-free DMEM with the relevant concentrations of Estradiol, Fulvestrant and Tamoxifen. Twenty-microlitre drops were dispensed on to a $10-\mathrm{cm}$ dish lid. Five millilitre of medium were added to the bottom of the dish to prevent evaporation. The lid was inverted and the drops were left hanging for 5 days. The newly formed organoids were then transferred into $10 \mu \mathrm{l}$ Matrigel drops (BD 356237, BD 356234) and moved to 24- or 48-well plates after which immunofluorescence or real-time monitoring (respectively) was carried out. Two hundred microlitre of medium with the relevant concentration of drugs were added to the wells.

Real-time invasion and migration assays. Cell invasion and migration were assessed in real time using the xCELLigence system CIM-16 wells plates. The bottom chamber was filled with $160 \mu \mathrm{l}$ of full medium (phenol-red or phenol-redfree DMEM with the relevant concentrations of Estradiol, Fulvestrant and Tamoxifen) and acted as a chemoattractant. The upper chamber was then secured on top and $30 \mu \mathrm{l}$ of serum-free medium were added to avoid the membrane drying out. The plate was loaded on the machine cradle and left for $1 \mathrm{~h}$ to allow membrane equilibration. After $1 \mathrm{~h}$, the baseline measurement was taken. Hundred microlitre of serum-free medium (again phenol-red or phenol-red-free DMEM with the relevant concentration of Estradiol, Fulvestrant and Tamoxifen) containing 40,000 cells were subsequently added to each well, and left for $30 \mathrm{~min}$ at room temperature to allow for the attachment of the cells. The impedance value for each well was recorded every $15 \mathrm{~min}$ for $20 \mathrm{~h}$ for migration experiments and $48 \mathrm{~h}$ for invasion and expressed as Cell Index value. For invasion, the CIM plates were coated with $30 \mu \mathrm{l}$ of a 1:40 dilution of Matrigel (BD Biosciences) and allowed to set for $4 \mathrm{~h}$ at $37^{\circ} \mathrm{C}$ before proceeding as per the migration experiment. Four wells per condition were used in each experiment and every experiment was repeated four times.

Chromatin immunoprecipitation (ChIP). Cells were crosslinked with $1 \%$ formaldehyde and processed according to Schmidt et al. ${ }^{55}$ Briefly, chromatin extract were sonicated using a Diagenode sonicator using 20 cycles $(30 \mathrm{~s}$ on and $30 \mathrm{~s}$ off) at maximum intensity. Purified chromatin was then immuno precipitated using $4 \mu \mathrm{g}$ of K27ac antibodies (Abcam ab4729) per ChIP. Nonimmunoprecipitated chromatin was used as Input control. Chromatin was then decrosslinked and sonication efficiencies tested on a $1.5 \%$ agarose gel. Before the construction of ChIP-seq libraries (NEB, see Supplementary Methods), we determined the enrichment using positive and negative controls. We then used $10 \mathrm{ng}$ of IPed chromatin and $10 \mathrm{ng}$ of Input for library preparation.

Bioinformatic analyses. Detailed protocols on Peak Calling, enhancers-promoters Peak-pairing, Clustering analysis, IPA analysis and RNA-seq differential expression analysis can be found in the Supplementary Methods.

RT-qPCR and ChIP-qPCR. RT-qPCR and ChIP-qPCR were performed and analysed as follows. Briefly, reactions were carried on in $10 \mu \mathrm{l}$ volume containing $5 \mu \mathrm{l}$ of SYBR mix (ABI 4472918), $0.5 \mu \mathrm{l}$ of primers (2.5 and $5 \mu \mathrm{M}$ final concentration, respectively), $2.5 \mu \mathrm{l}$ of complementary DNA or DNA and $2 \mu \mathrm{l}$ of water. We used a three-step cycle programme with melting analysis. The cycles were as follow: $10 \mathrm{~s}$ at $95^{\circ} \mathrm{C}, 30 \mathrm{~s}$ at $60^{\circ} \mathrm{C}$ and $30 \mathrm{~s}$ at $72^{\circ} \mathrm{C}$, repeated 40 times $^{7}$.

SRB and $\mathrm{IC}_{\mathbf{5 0}}$ assay. SRB and $\mathrm{IC}_{50}$ assays were performed as follow: briefly, the sulphorhodamine B (SRB) assay was used to monitor the effects of statin or Terbinafine treatment cell on cell proliferation in monolayer cultures. Cells were seeded in flat-bottomed 96 -well plates $\left(3 \times 10^{3}\right.$ cells per well). The cells were allowed to adhere overnight. One plate was assayed at this time point (day 0 ) and further plates were assayed at 2-day intervals. The cells were fixed by adding $100 \mu \mathrm{l}$ per well of ice-cold $40 \%$ (vol/vol) TCA to each well for $60 \mathrm{~min}$. The plates were washed five times in running tap water and stained with $100 \mu \mathrm{l}$ per well of the SRB reagent $(0.4 \% \mathrm{wt} / \mathrm{vol} \mathrm{SRB}$ in $1 \%(\mathrm{vol} / \mathrm{vol})$ acetic acid) for $30 \mathrm{~min}$. The plates were washed five times in $1 \%(\mathrm{vol} / \mathrm{vol})$ acetic acid and allowed to dry overnight. SRB was solubilized with $100 \mu \mathrm{l}$ per well $10 \mathrm{mM}$ Tris-base, shaken for $30 \mathrm{~min}$ and the optical density was measured at $492 \mathrm{~nm}$.

Primers. The sequences of primers (RT-qPCR, ChIP-qPCR) are listed in Supplementary Data 7.

\section{References}

1. Early Breast Cancer Trialists' Collaborative Group (EBCTCG) et al. Relevance of breast cancer hormone receptors and other factors to the efficacy of adjuvant tamoxifen: patient-level meta-analysis of randomised trials. Lancet 378, 771-784 (2011).

2. Palmieri, C., Patten, D. K., Januszewski, A., Zucchini, G. \& Howell, S. J. Breast cancer: current and future endocrine therapies. Mol. Cell. Endocrinol. 382, 695-723 (2014).

3. Musgrove, E. A. \& Sutherland, R. L. Biological determinants of endocrine resistance in breast cancer. Nat. Rev. Cancer, 1-13, doi:10.1038/nrc2713 (2009).

4. Xie, W. et al. Epigenomic analysis of multilineage differentiation of human embryonic stem cells. Cell 153, 1134-1148 (2013).

5. Consortium, T. E. P. et al. An integrated encyclopedia of DNA elements in the human genome. Nature 488, 57-74 (2012).

6. Polo, J. M. et al. A molecular roadmap of reprogramming somatic cells into iPS cells. Cell 151, 1617-1632 (2012).

7. Magnani, L. et al. Genome-wide reprogramming of the chromatin landscape underlies endocrine therapy resistance in breast cancer. Proc. Natl Acad. Sci. USA 110, E1490-E1499 (2013).

8. Sharma, S. V. et al. A chromatin-mediated reversible drug-tolerant state in cancer cell subpopulations. Cell 141, 69-80 (2010).

9. Suvà, M. L. et al. Reconstructing and reprogramming the tumor-propagating potentialof glioblastoma stem-like cells. Cell 157, 1-15 (2014).

10. Ernst, J. \& Kellis, M. Nat Biotechnol 2010 Ernst. Nat. Biotechnol. 28, 817-838 (2010).

11. Whyte, W. A. et al. Master transcription factors and mediator establish superenhancers at key cell identity genes. Cell 153, 307-319 (2013).

12. Heintzman, N. D. et al. Histone modifications at human enhancers reflect global cell-type-specific gene expression. Nature 459, 108-112 (2009).

13. Malik, A. N. et al. Genome-wide identification and characterization of functional neuronal activity-dependent enhancers. Nat. Neurosci. 17, 1330-1339 (2014).

14. Stasevich, T. J. et al. Regulation of RNA polymerase II activation by histone acetylation in single living cells. Nature 516, 272-275 (2014).

15. Shaw, L. E., Sadler, A. J., Pugazhendhi, D. \& Darbre, P. D. Changes in oestrogen receptor- $\alpha$ and $-\beta$ during progression to acquired resistance to tamoxifen and fulvestrant (Faslodex, ICI 182,780) in MCF7 human breast cancer cells. J. Steroid Biochem. Mol. Biol. 99, 19-32 (2006).

16. Martin, L. A. et al. An in vitro model showing adaptation to long-term oestrogen deprivation highlights the clinical potential for targeting kinase pathways in combination with aromatase inhibition. Steroids 76, 772-776 (2011).

17. Magnani, L., Ballantyne, E. B., Zhang, X. \& Lupien, M. PBX1 genomic pioneer function drives $E R \alpha$ signaling underlying progression in breast cancer. PLoS Genet. 7, e1002368 (2011).

18. Magnani, L. et al. The pioneer factor PBX1 is a novel driver of metastatic progression in ER $\alpha$-positive breast cancer. Oncotarget 6, 21878-21891 (2015).

19. Magnani, L., Eeckhoute, J. \& Lupien, M. Pioneer factors: directing transcriptional regulators within the chromatin environment. Trends Genet. 27, 465-474 (2011)

20. Magnani, L. \& Lupien, M. Chromatin and epigenetic determinants of estrogen receptor alpha (ESR1) signaling. Mol. Cell. Endocrinol. 382, 633-641 (2014).

21. Jozwik, K. M. \& Carroll, J. S. Pioneer factors in hormone-dependent cancers. Nat. Rev. Cancer 12, 381-385 (2012).

22. Tyson, J. J. et al. Dynamic modelling of oestrogen signalling and cell fate in breast cancer cells. Nat. Rev. Cancer 11, 523-532 (2011).

23. Zhang, Y. et al. Model-based analysis of ChIP-Seq (MACS). Genome Biol. 9, R137 (2008).

24. Miller, W. R. \& Larionov, A. Changes in expression of oestrogen regulated and proliferation genes with neoadjuvant treatment highlight heterogeneity of clinical resistance to the aromatase inhibitor, letrozole. Breast Cancer Res. 12, R52 (2010).

25. Bertucci, F. Gene expression profiling shows medullary breast cancer is a subgroup of basal breast cancers. Cancer Res. 66, 4636-4644 (2006).

26. Pasquali, L. et al. Pancreatic islet enhancer clusters enriched in type 2 diabetes risk-associated variants. Nat. Genet. 46, 136-143 (2014).

27. Hnisz, D. et al. Super-enhancers in the control of cell identity and disease. Cell 155, 934-947 (2013) 
28. Dowen, J. M. et al. Control of cell identity genes occurs in insulated neighborhoodsin mammalian chromosomes. Cell 159, 374-387 (2014).

29. Neph, S. et al. An expansive human regulatory lexicon encoded in transcription factor footprints. Nature 488, 83-90 (2012).

30. Jeon, T.-I. \& Osborne, T. F. SREBPs: metabolic integrators in physiology and metabolism. Trends Endocrinol Metab 23, 65-72 (2012).

31. Yokoyama, C. et al. SREBP-1, a basic-helix-loop-helix-leucine zipper protein that controls transcription of the low density lipoprotein receptor gene. Cell 75, 187-197 (1993).

32. Briggs, M. R., Yokoyama, C., Wang, X., Brown, M. S. \& Goldstein, J. L. Nuclear protein that binds sterol regulatory element of low density lipoprotein receptor promoter. I. Identification of the protein and delineation of its target nucleotide sequence. J. Biol. Chem. 268, 14490-14496 (1993).

33. Kamisuki, S. et al. A small molecule that blocks fat synthesis by inhibiting the activation of SREBP. Chem. Biol. 16, 882-892 (2009).

34. Nora, E. P. et al. Spatial partitioning of the regulatory landscape of the X-inactivation centre. Nature 485, 381-385 (2012).

35. Sanchez, C. G. et al. Preclinical modeling of combinedphosphatidylinositol-3kinase inhibition withendocrine therapy for estrogen receptor-positivebreast cancer. Breast Cancer Res. 13, R21 (2011).

36. Umetani, M. et al. 27-Hydroxycholesterol is an endogenous SERM that inhibits the cardiovascular effects of estrogen. Nat. Med. 13, 1185-1192 (2007).

37. Phimister, E. G., Warner, M. \& Gustafsson, J.-Å. On estrogen, cholesterol metabolism, and breast cancer. N. Engl. J. Med. 370, 572-573 (2014).

38. Carroll, J. S. et al. Chromosome-wide mapping of estrogen receptor binding reveals long-range regulation requiring the forkhead protein FoxA1. Cell 122, 33-43 (2005).

39. Wu, Q. et al. 27-Hydroxycholesterol promotescell-autonomous, ER-positive breast cancer growth. Cell Rep. 5, 637-645 (2013).

40. Lupien, M. et al. Growth factor stimulation induces a distinct ER cistrome underlying breast cancer endocrine resistance. Genes Dev. 24, 2219-2227 (2010).

41. Greaves, M. \& Maley, C. C. Clonal evolution in cancer. Nature 481, 306-313 (2012).

42. Freed-Pastor, W. A. et al. Mutant p53 disrupts mammary tissue architecture via the mevalonate pathway. Cell 148, 244-258 (2012).

43. Miller, W. R. et al. Gene expression profiles differentiating between breast cancers clinically responsive or resistant to Letrozole. J. Clin. Oncol. 27, 1382-1387 (2009).

44. Parris, T. Z. et al. Frequent MYC coamplification and DNA hypomethylation ofmultiple genes on 8q in 8p11-p12-amplified breast carcinomas. Oncogenesis 3, e95-e98 (2014).

45. Yamaguchi, N. et al. FoxA1 as a lineage-specific oncogene in luminal type breast cancer. Biochem. Biophys. Res. Commun. 365, 711-717 (2008).

46. Lin, M.-L. et al. Expression profiling of nuclear receptors in breast cancer identifies TLX as a mediator of growth and invasion in triple-negative breast cancer. Oncotarget 6, 21685-21703 (2015).

47. Ahern, T. P., Lash, T. L., Damkier, P., Christiansen, P. M. \& Cronin-Fenton, D. P. Statins and breast cancer prognosis: evidence and opportunities. Lancet Oncol. 15, e461-e468 (2014).

48. Ahern, T. P. et al. Statin prescriptions and breast cancer recurrence risk: a Danish Nationwide Prospective Cohort Study. J. Natl Cancer Inst. 103, 1461-1468 (2011)

49. Pagani, O. et al. Adjuvant exemestane with ovarian suppression in premenopausal breast cancer. N. Engl. J. Med. 371, 107-118 (2014).

50. Bao, T., Blackford, A. L. \& Stearns, V. Effect of simvastatin on the pharmacokinetics of anastrozole. Breast Cancer Res. Treat. 131, 709-711 (2011)
51. Kobayashi, Y. et al. Mevalonate pathway antagonist inhibits proliferation of serous tubal intraepithelial carcinoma and ovarian carcinoma in mouse models. Clin. Cancer Res. doi: 10.1158/1078-0432.CCR-14-3368 (2015).

52. Clendening, J. W. et al. Exploiting the mevalonate pathway to distinguish statin-sensitive multiple myeloma. Blood 115, 4787-4797 (2010).

53. Lombardo, Y. et al. Nicastrin regulates breast cancer stem cell properties and tumor growth in vitro and in vivo. Proc. Natl Acad. Sci. USA 109, 16558-16563 (2012).

54. Warita, K. et al. Statin-induced mevalonate pathway inhibition attenuates the growth of mesenchymal-like cancer cells that lack functional E-cadherin mediated cell cohesion. Sci. Rep. 4, 7593 (2014).

55. Schmidt, D. et al. ChIP-seq: using high-throughput sequencing to discover protein-DNA interactions. Methods 48, 240-248 (2009).

\section{Acknowledgements}

This work was supported by an Imperial Junior Research Fellowship to L.M. and a Wellcome Trust grant (103034/Z/13/Z) to D.K.P. and the OTKA K108655 grant to B.G. We wish to thank Mrs Lorna Watson for her help with the manuscript. The Imperial College Healthcare NHS Trust Tissue Bank provided tissue samples. Other investigators may have received samples from these same tissues. The research was supported by the National Institute for Health Research (NIHR) Biomedical Research Centre, the Experimental Cancer Medicine Centre and the Cancer Research UK Centre at Imperial College Healthcare NHS Trust, Imperial College, London. The views expressed are those of the author(s) and not necessarily those of the NHS, the NIHR or the Department of Health.

\section{Author contributions}

L.M. designed the study. L.M., I.B., B.G., S.M., L.C. and R.C.C. carried out the bioinformatic analysis and interpreted the data. L.M. wrote the manuscript. V.T.M.N., M.F., Y.L., D.K.P., G.F., P.D., A.M. and J.H.S. carried out laboratory experiments. R.C.C., L.W and N.P. recruited patients, collected and prepared clinical specimens.

\section{Additional information}

Accession codes: RNA-seq can be accessed at ENA (http://www.ebi.ac.uk/ena) with the code PRJEB11448. ChIP-seq data can be found in the GEO archive under number GSE60517.

Supplementary Information accompanies this paper at http://www.nature.com/ naturecommunications

Competing financial interests: The authors declare no competing financial interests.

Reprints and permission information is available online at http://npg.nature.com/ reprintsandpermissions/

How to cite this article: Nguyen, V. T. M. et al. Differential epigenetic reprogramming in response to specific endocrine therapies promotes cholesterol biosynthesis and cellular invasion. Nat. Commun. 6:10044 doi: 10.1038/ncomms10044 (2015).

This work is licensed under a Creative Commons Attribution 4.0 International License. The images or other third party material in this article are included in the article's Creative Commons license, unless indicated otherwise in the credit line; if the material is not included under the Creative Commons license, users will need to obtain permission from the license holder to reproduce the material To view a copy of this license, visit http://creativecommons.org/licenses/by/4.0/ 


\title{
Author Correction: Differential epigenetic reprogramming in response to specific endocrine therapies promotes cholesterol biosynthesis and cellular invasion
}

\author{
Van T.M. Nguyen ${ }^{1,8}$, Iros Barozzi ${ }^{2,8}$, Monica Faronato ${ }^{1}, Y_{\text {Ylenia Lombardo }}{ }^{1}$, Jennifer H. Steel ${ }^{1}$, Naina Patel ${ }^{1}$, \\ Philippa Darbre 3 , Leandro Castellano1, Balázs Győrffy 4,5, Laura Woodley6 ${ }^{6}$ Alba Rodriguez-Meira1, \\ Darren K. Patten ${ }^{1}$, Valentina Vircillo ${ }^{7}$, Manikandan Periyasamy ${ }^{1}$, Simak Ali ${ }^{1}$, Gianmaria Frige ${ }^{2}$, Saverio Minucci ${ }^{2}$, \\ R. Charles Coombes ${ }^{1} \&$ Luca Magnani $^{1}$
}

Correction to: Nature Communications https://doi.org/10.1038/ncomms10044, published online 27 November 2015

This Article omits a declaration from the Competing Interests statement, which should have included the following: 'One of the authors, Y.L., is an editor on the staff of Nature Communications, but was not in any way involved in the journal review process. The other authors declare no competing interests.'

Also, the Article contains an error in the name of the author Alba Rodriguez-Meira, which is incorrectly given as Alba Meira.

These errors have not been corrected in either the PDF or HTML versions of the Article.

Published online: 06 August 2019

\begin{abstract}
(c) (i) Open Access This article is licensed under a Creative Commons Attribution 4.0 International License, which permits use, sharing, adaptation, distribution and reproduction in any medium or format, as long as you give appropriate credit to the original author(s) and the source, provide a link to the Creative Commons license, and indicate if changes were made. The images or other third party material in this article are included in the article's Creative Commons license, unless indicated otherwise in a credit line to the material. If material is not included in the article's Creative Commons license and your intended use is not permitted by statutory regulation or exceeds the permitted use, you will need to obtain permission directly from the copyright holder. To view a copy of this license, visit http://creativecommons.org/licenses/by/4.0/.
\end{abstract}

(c) The Author(s) 2019

\footnotetext{
${ }^{1}$ Department of Surgery and Cancer, Imperial College London, London W12 ONN, UK. ${ }^{2}$ IFOM-IEO Campus, European Institute of Oncology, Milan 20139 , Italy. ${ }^{3}$ School of Biological Science, University of Reading, Reading RG6 6LA, UK. ${ }^{4}$ MTATTK Lendület Cancer Biomarker Research Group, 2nd Department of Pediatrics, Semmelweis University, Budapest H-1117, Hungary. ${ }^{5}$ MTA-SE Pediatrics and Nephrology Research Group, 2nd Department of Pediatrics,

Semmelweis University, Budapest H-1117, Hungary. ${ }^{6}$ ECMC, Charing Cross Hospital, London W120nn, UK. 7 Department of Pharmacy, Health and Nutritional Sciences, University of Calabria, Arcavacata di Rende (CS) 87036, Italy. ${ }^{8}$ These authors contributed equally: Van T. M. Nguyen, Iros Barozzi. Correspondence and requests for materials should be addressed to L.M. (email: I.magnani@imperial.ac.uk)
} 